\title{
SCATTERING OF IRREGULAR WAVES BY VERTICAL CYLINDERS
}

\author{
WOO SUN PARK \\ Coastal and Harbor Engineering Research Center, \\ Korea Ocean Research and Development Institute, \\ Ansan P.O. Box 29, Seoul 425-600, Korea \\ BUM HYOUNG KIM \\ Hanah Engineering Co., Ltd., Kwansesa Bldg. 4Fl., 209-9 Nonhyun-Dong, \\ Kangnam-Ku, Seoul 135-010, Korea
}

KYUNG DOUG SUH and KIL SEONG LEE

School of Civil, Urban and Geo-Systems Engineering, Seoul National University,

Seoul 151-742, Korea 


\begin{abstract}
An analytical model has been developed that can predict the scattering of irregular waves normally incident upon an array of vertical cylinders. To examine the predictability of the developed model, laboratory experiments have been made for the reflection and transmission of irregular waves from arrays of circular cylinders with various diameters and gap widths. Though the overall agreement between measurement and calculation is fairly good, the model tends to over- and underpredict the reflection and transmission coefficients, respectively, as the gap width decreases. The model also underestimates the energy loss coefficients for small gap widths because it neglects the evanescent waves near the cylinders. The peaks of the measured spectra of the reflected and transmitted waves slightly shift towards higher frequencies compared with that of the incident wave spectrum probably because of the generation of shorter period waves due to the interference of the cylinders. Both model and experimental data show that the wave reflection and transmission become larger and smaller, respectively, as the wave steepness increases, which is a desirable feature of the cylinder breakwaters.
\end{abstract}

Keywords: Analytical models, breakwaters, cylinders, irregular waves, wave scattering 


\section{Introduction}

Slotted breakwaters made of concrete units or timbers have gained popularity in many countries where a source of gravel needed for the rubble mound breakwater is scarce. They have some other desirable features that have encouraged their use within harbors. Not only do they offer protection behind the breakwater but they reduce wave reflection from the breakwater, which can sometimes cause difficulties in navigation or ship operation (see McBride et al., 1994, for example). In addition they permit the circulation of water and so assist the maintenance of the water quality within the harbor.

The simplest structure of a slotted breakwater is an array of vertical cylinders. The closely spaced cylinders induce energy dissipation due to the viscous eddies formed by the flow through the gaps. The functional efficiency of the cylinder breakwater is evaluated by examining the reflection and transmission of the waves from the breakwater. In order to examine the wave scattering by vertical cylinders, hydraulic model tests have been used (Hayashi et al., 1966; Kojima et al., 1988; Uda et al., 1990; Kakuno and Liu, 1993, among others). Efforts towards developing analytical models for predicting the reflection and transmission coefficients have also been made. Twersky (1962), Spring and Monkmeyer (1974) and Linton and Evans (1990) provided analytical solutions for circular cylinders. For cylinders with an arbitrary cross-section, analytical models were developed based on the method of matched asymptotic expansions (Lamb, 1932; Martin and Dalrymple; 1988; Kakuno and Liu, 1993). Other authors (Hagiwara, 1984; Bennett et al., 1992) used an eigenfunction expansion method to solve the boundary value problem derived from the linear description of the water wave system diffracted by the cylinders. Among the above-mentioned analytical models, only the last three authors included the energy dissipation by flow separation around the cylinders, which becomes important for closely spaced cylinders. Others only took the diffraction problem into consideration by assuming that the spacing is much greater than the diameter of the cylinders.

The aforementioned experimental or analytical approaches deal with regular waves impinging on vertical cylinders. In the present study, using an eigenfunction expansion method, we develop an analytical model that can predict the reflection and transmission of irregular waves normally incident upon vertical cylinders. In order to examine the performance of the developed model, laboratory experiments are made for circular cylinders in a wave flume. The comparisons between the 
model and experimental results are made for the frequency-averaged reflection and transmission coefficients as well as those of the individual frequency components.

\section{Theoretical Analysis}

Let us consider the array of vertical cylinders of an arbitrary cross-section sketched in Fig. 1, in which $h$ is the constant water depth in still water. The distance between the centers of two adjacent cylinders is denoted as $2 \mathrm{~A}$ and the width of an opening is $2 a$ so that the porosity of the cylinder breakwater at $x=0$ is defined as $r_{0}=a / A$. The $x$-axis and $y$-axis are taken to be normal and parallel, respectively, to the crest line of the cylinders. The vertical coordinate $z$ is measured vertically upwards from the still water line.

Consider unidirectional irregular waves incident in the positive $x$-direction. Based on the small-amplitude wave theory, the surface elevation of the incident waves can be expressed as

$$
\eta_{I}(x, t)=\sum_{n=1}^{\infty} \frac{H_{n}}{2} e^{i\left(k_{n} x-\omega_{n} t+\varepsilon_{n}\right)}
$$

in which $i=\sqrt{-1}$, and $H_{n}, k_{n}$ and $\varepsilon_{n}$ are the height, wave number and phase angle, respectively, of the $n$th component wave whose angular frequency is $\omega_{n}$. The subscript $I$ denotes the incident waves. The wave number $k_{n}$ must satisfy the dispersion relationship:

$$
\omega_{n}^{2}=g k_{n} \mathrm{ta} \mathrm{n} k_{k}(h)
$$

in which $g$ is the gravitational acceleration.

The velocity potential consists of free propagating wave modes and nonpropagating evanescent wave modes. We assume the 'wide spacing approximation' (Srokosz and Evans, 1979) so that the evanescent waves near the cylinders may be neglected. The total velocity potential for the propagating wave modes $\Phi^{\prime}(x, z, t)$ can be expressed as 


$$
\Phi^{\prime}(x, z, t)=\sum_{n=1}^{\infty} \Phi_{n}(x, z, t)=\sum_{n=1}^{\infty} \frac{g H_{n}}{2 \omega_{n}} \phi_{n}(x) \frac{\cos \mathbf{h}_{n}(z+h)}{\cos \mathbf{k}_{n} h} e^{i\left(-\omega_{n} t+\varepsilon_{n}\right)}
$$

in which $\phi_{n}(x)$ is the horizontal spatial variation of the $n$th component wave potential $\Phi_{n}$.

Very near the cylinders the waves may exhibit three-dimensional features. However, in the region far from the cylinders, the waves may become long-crested. Therefore, the solutions for $\phi_{n}(x)$ in each region of the fluid domain sketched in Fig. 1 may be constructed as

$$
\begin{array}{ll}
\phi_{n 1}(x)=e^{i k_{n} x}+K_{R n} e^{-i k_{n} x}, & x \leq 0 \\
\phi_{n 2}(x)=K_{T n} e^{i k_{n} x}, & x \geq 0
\end{array}
$$

in which $K_{R n}$ and $K_{T n}$ are the complex-valued reflection and transmission coefficients, respectively, of the $n$th component wave. The subscripts 1 and 2 represent Region $1(x \leq 0)$ and $2(x \geq 0)$, respectively, in Fig. 1.

The potential $\phi_{n j}(x)(j=1,2)$ must satisfy the matching conditions at the location of the cylinders (i.e. $x=0$ ) which provide continuity of pressure and horizontal velocity normal to the vertical plane separating the fluid regions, i.e.

$$
\begin{aligned}
& \phi_{n 2}=\phi_{n 1}+2 C \frac{d \phi_{n 1}}{d x}+\frac{i \bar{\beta}}{\omega_{n}} \frac{d \phi_{n 1}}{d x} \text { as }|x| \rightarrow 0 \\
& \frac{d \phi_{n 1}}{d x}=\frac{d \phi_{n 2}}{d x} \text { as }|x| \rightarrow 0
\end{aligned}
$$

in which $C$ is the blockage coefficient and $\bar{\beta}$ is the depth-averaged linearized dissipation coefficient which will be derived later in this paper. See Kakuno and Oda (1986) and Kakuno and Liu (1993) for the blockage coefficients for various shapes of cylinders. The second and third terms in (6) represent inertia resistance and energy dissipation due to flow separation near the cylinders, respectively. For the derivation of (6), see Kakuno and Liu (1993). They derived this equation for regular waves. Since the blockage coefficient $C$ depends only on the geometry of 
the cylinder array but not on the wave characteristics, the inertia term can be used without change for irregular waves. However, the energy dissipation term for regular waves should be different from that for irregular waves, which will be derived later in this paper. The matching conditions (6) and (7) provide the boundary conditions for the far-field solutions on two sides of the cylinder array. Since the near-field length scale is of the order of wave amplitude, which is much smaller than the far-field length scale of $O\left(k^{-1}\right)$, these matching conditions can be approximately applied to the far-field solutions by letting $x \rightarrow \pm 0$.

The reflection and transmission coefficients of each component wave can be derived as follows. Let us express them as

$$
\begin{aligned}
& K_{R n}=a_{0}+i b_{0} \\
& K_{T n}=c_{0}+i d_{0}
\end{aligned}
$$

Substituting these expressions into (4) and (5) and applying the matching conditions (6) and (7), we obtain

$$
\begin{aligned}
& a_{0}+c_{0}=1 \\
& b_{0}+d_{0}=0 \\
& \left(1+R_{n}\right) a_{0}+P_{n} b_{0}-c_{0}=R_{n}-1 \\
& P_{n} a_{0}-\left(1+R_{n}\right) b_{0}+d_{0}=P_{n}
\end{aligned}
$$

in which $P_{n}=2 C k_{n}$ and $R_{n}=\bar{\beta} k_{n} / \omega_{n}$. Solving these equations for $a_{0}$ to $d_{0}$ and substituting them into (8) and (9), we get

$$
\begin{aligned}
& K_{R n}=\frac{R_{n}\left(R_{n}+2\right)+P_{n}^{2}}{\left(R_{n}+2\right)^{2}+P_{n}^{2}}-\frac{2 P_{n}}{\left(R_{n}+2\right)^{2}+P_{n}^{2}} i \\
& K_{T n}=\frac{2\left(R_{n}+2\right)}{\left(R_{n}+2\right)^{2}+P_{n}^{2}}+\frac{2 P_{n}}{\left(R_{n}+2\right)^{2}+P_{n}^{2}} i
\end{aligned}
$$

The remaining task is to determine $\bar{\beta}$ for an array of vertical cylinders. The linearized dissipation coefficient $\bar{\beta}$, which represents the energy loss due to flow separation, may actually be dependent upon the wave frequency. However, the 
flow separation due to irregular waves is induced not by the individual component waves but by the superposition of all the component waves. Therefore, we seek the dissipation coefficient $\bar{\beta}$, which corresponds to the total wave. By applying global momentum conservation to a control volume in the vicinity of the cylinder gap, Mei (1983, p. 257) obtained the dynamic matching condition for the total pressure as

$$
\frac{p_{2}}{\rho}-\frac{p_{1}}{\rho}+2 C \frac{\partial u_{1}}{\partial t}+\frac{\alpha}{2} u_{1}\left|u_{1}\right|=0
$$

in which $p=-\rho \partial \Phi^{\prime} / \partial t$ is the dynamic wave pressure, $\rho$ is the fluid density, $u_{1}=\partial \Phi^{\prime}{ }_{1} / \partial x$ is the wave-induced velocity away from the cylinders, and $\alpha$ is the energy loss coefficient at the gap. Note that the preceding equation is equivalent to (6) if the nonlinear energy dissipation term is linearized by $(\alpha / 2)\left|u_{1}\right| u_{1}=\bar{\beta} u_{1}$

For rectangular cylinders, the energy loss coefficient $\alpha$ has been evaluated using the plate orifice formula (e.g., Kondo, 1979):

$$
\alpha=\left(\frac{1}{r_{0} C_{c}}-1\right)^{2}
$$

in which $C_{c}$ is the empirical contraction coefficient at the gap. For circular cylinders, however, this formula could not be used as it is because the porosity changes along the gap, i.e., $r=r(x)$. Noting that the energy loss coefficient is approximately proportional to the reciprocal of the square of the porosity, we introduce an ad hoc porosity $\bar{r}$ so that

$$
\frac{1}{r^{2}}=\frac{1}{d} \int_{-d / 2}^{d / 2} \frac{d x}{r(x)^{2}}
$$

in which $d$ is the diameter of the circular cylinder and $r(x)$ is given by 


$$
r(x)=1-\frac{\sqrt{\left(\frac{d}{2}\right)^{2}-x^{2}}}{\frac{d}{2}+a}
$$

The energy loss coefficient for circular cylinders is then evaluated by

$$
\alpha=\left(\frac{1}{\bar{r} C_{c}}-1\right)^{2}
$$

Hattori (1972) concluded that the contraction coefficient $C_{c}$ ranged from 0.4 to 0.75 for rectangular cylinders, but our experimental data show that $C_{c}=0.8$ is appropriate for circular cylinders as will be discussed in the next section.

The energy dissipation term due to flow separation, i.e., the fourth term in (16), is nonlinear. It can be linearized by approximating it to

$$
\frac{\alpha}{2} u_{1}\left|u_{1}\right| \approx \beta(z) u_{1}
$$

in which $\beta(z)$ is the depth-dependent linearized dissipation coefficient. If we define the error involved in the approximation of (21) as $\delta$ :

$$
\delta=\left(\beta(z)-\frac{\alpha}{2}\left|u_{1}\right|\right) u_{1}
$$

the expression for $\beta(z)$ can be obtained by minimizing the expectation of the squared error $\delta^{2}$ with respect to $\beta(z)$, i.e. $E\left[\partial \delta^{2} / \partial \beta(z)\right]=0$, as

$$
\beta(z)=\frac{\alpha}{2} \frac{E\left[\left|u_{1}\right|^{3}\right]}{E\left[u_{1}^{2}\right]}
$$

Assuming that $u_{1}$ is a Gaussian random process with zero mean and the standard deviation of $\sigma(z), \beta(z)$ is given by 


$$
\beta(z)=\frac{\alpha}{2} \sqrt{\frac{8}{\pi}} \sigma(z)
$$

The linearized dissipation coefficient is still a function of the depth. The depthaveraged dissipation coefficient, $\bar{\beta}$, can be obtained by equating the expectations of the depth-integrated powers, i.e.

$$
\bar{\beta} E\left[\int_{-h}^{0} u_{1}^{2} d z\right]=E\left[\int_{-h}^{0} \beta(z) u_{1}^{2} d z\right]
$$

Using (24) and $E\left[u_{1}^{2}\right]=\sigma(z)^{2}, \bar{\beta}$ is finally given by

$$
\bar{\beta}=\frac{\alpha}{2} \sqrt{\frac{8}{\pi}} \frac{\int_{-h}^{0} \sigma(z)^{3} d z}{\int_{-h}^{0} \sigma(z)^{2} d z}
$$

The Gaussian quadrature formulas can be used for the integration over the depth in the preceding equation.

The remaining task for calculating $\bar{\beta}$ is to express the standard deviation $\sigma(z)$ in terms of the incident wave spectrum. The amplitude of $u_{n 1}\left(=\partial \Phi_{n 1} / \partial x\right)$, $U_{n 1}$, can be obtained by substituting $K_{R n}$ given by (14) into (4) as

$$
U_{n 1}=\frac{H_{n}}{2} T_{n}
$$

in which $T_{n}$ is given by

$$
T_{n}=\omega_{n} \frac{\cos \mathbf{k}_{n}(z+h)}{\sin \mathbf{k}_{n} h} \frac{2}{\sqrt{\left(R_{n}+2\right)^{2}+P_{n}^{2}}}
$$

Then the velocity spectrum $S_{u 1}\left(\omega_{n}\right)$ is related to the incident wave energy spectrum $S_{\eta_{I}}\left(\omega_{n}\right)$ by 


$$
S_{u 1}\left(\omega_{n}\right)=\left|T_{n}\right|^{2} S_{\eta_{l}}\left(\omega_{n}\right)
$$

The standard deviation of $u_{1}$ is then given in terms of continuous spectrum by

$$
\sigma(z)=\sqrt{\int_{0}^{\infty} S_{u 1}(\omega) d \omega}=\sqrt{\int_{0}^{\infty}|T(\omega)|^{2} S_{\eta_{I}}(\omega) d \omega}
$$

in which $T(\omega)$ is the transfer function given by (28) with the replacement of $\omega_{n}$ by $\omega$. The depth-averaged linearized dissipation coefficient $\bar{\beta}$ is then calculated by substituting (30) into (26). In (30) $T(\omega)$ is a function of $R_{n}$ as shown in (28), which is a function of $\bar{\beta}$. Therefore, (26) is a transcendental function of $\bar{\beta}$. To solve for $\bar{\beta}$, the initial value of $\bar{\beta}=0$ is used in (30) to calculate $\sigma(z)$, which is then substituted into (26) to calculate the new $\bar{\beta}$. Thus newly calculated $\bar{\beta}$ is then used in (30) to calculate $\sigma(z)$ again. The iteration was continued until the relative error between the new and old values of $\bar{\beta}$ is less than $0.001 \%$. The solution converges within less than ten iterations.

Once $\bar{\beta}$ is calculated, $K_{R n}$ and $K_{T n}$ can be calculated by (14) and (15), respectively, and then the spectral densities of the reflected and transmitted waves are calculated by

$$
\begin{aligned}
& S_{\eta_{R}}\left(\omega_{n}\right)=\left|K_{R n}\right|^{2} S_{\eta_{I}}\left(\omega_{n}\right) \\
& S_{\eta_{T}}\left(\omega_{n}\right)=\left|K_{T n}\right|^{2} S_{\eta_{I}}\left(\omega_{n}\right)
\end{aligned}
$$

respectively. Now $K_{R n}$ and $K_{T n}$ are understood as the transfer functions between the incident waves and the reflected or transmitted waves, respectively. The frequency-averaged reflection and transmission coefficients of the irregular 
waves are calculated by (Goda, 1985)

$$
\begin{aligned}
& K_{R}=\sqrt{\frac{m_{0}^{R}}{m_{0}^{I}}} \\
& K_{T}=\sqrt{\frac{m_{0}^{T}}{m_{0}^{I}}}
\end{aligned}
$$

in which $m_{0}^{I}, m_{0}^{R}$, and $m_{0}^{T}$ are the zeroth moments of the incident, reflected, and transmitted wave spectra, respectively, which are obtained by integrating each spectrum over the entire frequency range.

\section{Laboratory Experiment}

Experiments were carried out in the wave flume at the Coastal and Harbor Engineering Research Center of the Korea Ocean Research and Development Institute. The flume is $53 \mathrm{~m}$ long, $1.25 \mathrm{~m}$ high, and $1 \mathrm{~m}$ wide. It is equipped with a piston-type random wave generator at one end and a wave-absorbing beach at the other end. The wave generation and data acquisition are controlled by a personal computer. The wave-maker control system contains the so-called reflected wave absorbing filter that can suppress the re-reflection at the wave paddle of the waves reflected from the experimental structure. This is achieved by continuously sensing the reflected waves by a wave gauge attached at the front face of the paddle and correcting the input signal for the movement of the paddle. This makes it possible to carry out an irregular wave test for a long time without the accumulation of wave energy in the flume. Water surface displacement was measured with resistance-type wave gauges.

All the experiments were carried out in water of $50 \mathrm{~cm}$ depth. Two different diameters of poly-vinyl-chrolide pipes were used with various gap widths. The geometric parameters of the arrays of the cylinders used in the experiment and the corresponding coefficients are given in Table 1 , in which $\alpha$ is the energy loss coefficient calculated by (20) with $C_{c}=0.8$ and $C$ is the blockage coefficient calculated for circular cylinders by 


$$
C=\frac{A}{2} r_{0}^{-0.9}
$$

This formula was proposed by Kim (1998) based on Kakuno and Oda's (1986) series solutions. Numerical tests have been made for different values of $C_{c}$ varying from 0.6 to 1.0 at an increment of 0.1 , and the calculation with $C_{c}=0.8$ gave the best agreement with the measurement.

The incident wave spectrum used for the experiment was the BretschneiderMitsuyasu spectrum given by

$$
S_{\eta_{I}}(f)=0.205 H_{s}^{2} T_{s}\left(T_{s} f\right)^{-5} \exp \left[-0.75\left(T_{s} f\right)^{-4}\right]
$$

in which $f$ is the wave frequency and $H_{s}$ and $T_{s}$ are the significant wave height and period, respectively. The significant wave period varied from 1.0 to 2.0 $\mathrm{s}$ at the intervals of $0.2 \mathrm{~s}$. The significant wave height $H_{s} \approx 5 \mathrm{~cm}$ was used for $T_{s} \approx 1.0$ and $1.2 \mathrm{~s}$, and $H_{s} \approx 5$ and $10 \mathrm{~cm}$ were used for the longer wave periods.

In order to measure the incident, reflected, and transmitted wave spectra, four wave gauges were installed as shown in Fig. 2. The wave gauges W1 to W3 in front of the cylinders are for measuring the incident and reflected waves, and the gauge W4 behind the cylinders is for transmitted waves. The wave records measured at W1 to W3 were used to separate the incident and reflected wave spectra using the technique developed by Park et al. (1992). The wave measurements were made for 120 times the significant wave period at the sampling rate of $20 \mathrm{~Hz}$ for each of the wave gauges. A sufficient waiting time was allowed to elapse after the initiation of wave generation prior to data acquisition to permit the slower-traveling high-frequency component waves to travel to the remote wave gauge $\mathrm{W} 4$.

In the spectral analysis of the data, the first 2,048 data points were used for the waves of $T_{s} \approx 1.0$ to $1.6 \mathrm{~s}$, and 4,096 points for the waves of $T_{s} \approx 1.8$ and $2.0 \mathrm{~s}$. The time series was corrected by applying a $10 \%$ cosine taper on both ends and was subjected to spectral analysis. The raw spectrum was running-averaged twice over fifteen neighboring frequency bands, the total number of degrees of freedom of the final estimates being 225 .

\section{Results}


The comparison between measurement and calculation of the frequencyaveraged reflection and transmission coefficients is given in Table 2, in which the relative error was calculated by

$$
\text { Error }=\frac{K_{R c}-K_{R m}}{K_{R c}} \times 100 \% \quad \text { or } \quad \frac{K_{T c}-K_{T m}}{K_{T c}} \times 100 \%
$$

where the subscripts $c$ and $m$ denote calculation and measurement, respectively.

The comparison of the measured and calculated reflection and transmission coefficients is shown in Fig. 3. The correlation coefficients for reflection and transmission are 0.973 and 0.991 , respectively. Though the overall agreement is acceptable, the model tends to over-predict the reflection coefficients for larger values of them, which correspond to smaller gap widths. Likewise, the model tends to slightly under-predict the transmission coefficients for their smaller values, which again correspond to smaller gap widths.

To see in detail the discrepancy between measurement and calculation depending on the gap width, $K_{R c} / K_{R m}$ and $K_{T c} / K_{T m}$ are plotted as a function of the porosity in Figs. 4 and 5, respectively. Fig. 4 clearly shows that for the larger cylinders $(d=11.5 \mathrm{~cm})$ the model tends to under-predict the reflection coefficient as the porosity increases. For the smaller cylinders $(d=4.8 \mathrm{~cm})$, except for the cases of $H_{s}=5 \mathrm{~cm}$ and $r_{0}=0.417$ (the largest porosity in this experiment), a similar trend is observed even though it is not so clear as that for the larger cylinders. Fig. 5 shows that in opposition to the reflection coefficient the model tends to under-predict the transmission coefficient as the porosity decreases.

Another important parameter for examining the performance of the model is the energy loss coefficient $K_{L}$, which is related to the reflection and transmission coefficients by

$$
K_{L}=1-\left(K_{R}^{2}+K_{T}^{2}\right)
$$

In Fig. 6 are shown the values of $K_{L c} / K_{L m}$ as a function of the porosity, in which again the subscripts $c$ and $m$ denote calculation and measurement, respectively. Except for the cases of the largest porosity $\left(r_{0}=0.417\right)$, for which an exceptional behavior was shown also for the reflection coefficient [see Fig. 4 (a)], the calculated energy loss becomes less than the measured one as the porosity 
decreases. This discrepancy may be attributed to the assumption of 'wide spacing approximation' which neglects the evanescent waves near the cylinders. The evanescent waves may increase the energy loss through the gap between the cylinders, and their effects may increase with decreasing porosity. Therefore, the present model neglecting the evanescent waves may underestimate the energy loss coefficients for small gap widths.

To see the effect of the wave steepness, the reflection coefficients of the steep waves $\left(H_{s}=10 \mathrm{~cm}\right)$ against those of the mild waves $\left(H_{s}=5 \mathrm{~cm}\right)$ are plotted as a function of the porosity in Fig. 7 for both measurement and calculation. A similar plot is shown in Fig. 8 for the transmission coefficients. In general both measurement and calculation show larger reflection and smaller transmission for steeper waves except for the measured reflection coefficients in Cases 1 to 3 . Therefore the cylinder breakwater is more effective for steeper waves in protecting the harbor area, and it is more effective for milder waves in reducing the wave reflection from the breakwater. This feature is a desirable one because the harbor protection is important during the severe storms of steep waves while reducing wave reflection from the breakwater is important for navigation and ship operation during the ordinary conditions of mild swells.

To see if the gap width affects the calculation of the scattering of particular frequency bands of the irregular waves, the measured and calculated spectra of reflected and transmitted waves are plotted in Figs. 9 to 11 along with the measured incident spectrum for different gap widths but the same incident waves ( $T_{s} \approx 1.6 \mathrm{~s}$ and $H_{s} \approx 5 \mathrm{~cm}$ ). In these and the following figures, the thick and thin lines indicate the measurement and calculation, respectively. Note that the measured incident wave spectrum was used as the incident spectrum for the calculation. No particular difference depending on the gap width is observed. Instead it is observed that the peaks of the calculated spectra of the reflected and transmitted waves coincide with that of the incident wave spectrum but those of the measured spectra slightly shift towards higher frequencies. This seems to be attributed to the interference of the cylinders which often generates the waves with periods shorter than the incident wave period. Further investigation is needed to clarify this phenomenon.

To examine the influence of wave steepness on the performance of the model for different frequencies, the result for steeper waves $\left(H_{s} \approx 10 \mathrm{~cm}\right)$ is presented in Fig. 12, which should be compared with Fig. 10 having the same configuration of the cylinders and wave period but a smaller wave height $\left(H_{s} \approx 5 \mathrm{~cm}\right)$. For 
steeper waves, the reflection and transmission are over-predicted and underpredicted, respectively, by the model over the entire frequency range except for very low frequencies. The same trend has been observed for the frequencyaveraged reflection and transmission coefficients.

Figs. 13 and 14 along with Fig. 10 show the comparison among different significant wave periods for the same wave height and configuration of the cylinders. In these cases, the calculation errors for the frequency-averaged reflection or transmission coefficient are relatively small, ranging from -2.7 to $4.6 \%$ (see Table 2). Again no particular difference depending on the significant wave period is observed, but the errors for the individual frequency components are somewhat larger than the frequency-averaged errors in certain frequency ranges. For example, the calculation error for the frequency-averaged transmission coefficient is only $-2.7 \%$ in the case shown in Fig. 13, but the transmission of the individual frequency components is somewhat over-predicted for the lower frequencies and under-predicted for the higher frequencies. The same trend is observed in all the cases with different significant wave periods. This may again be attributed to the generation of shorter period waves due to the interference of the cylinders.

Finally we present the spectra for the cases of the best and worst comparison of the frequency-averaged reflection and transmission coefficients between measurement and calculation. Fig. 15 shows the results for Case 2 with $T_{s} \approx 1.0 \mathrm{~s}$ and $H_{s} \approx 5 \mathrm{~cm}$, for which the calculation errors for the frequency-averaged reflection and transmission coefficients are $0.8 \%$ and $-0.3 \%$, respectively. The overall agreement is very good for the transmitted waves, but it is not very good for the reflected waves even though the frequency-averaged reflection coefficients of measurement and calculation are almost identical. Fig. 16 shows the results for Case 5 with $T_{s} \approx 1.4 \mathrm{~s}$ and $H_{s} \approx 10 \mathrm{~cm}$, for which the calculation errors for the frequency-averaged reflection and transmission coefficients are $15.7 \%$ and $-25.1 \%$, respectively. The reflection and transmission are overestimated and underestimated, respectively, by the analytical model over the entire frequency band.

\section{Conclusions}

Using an eigenfunction expansion method, an analytical model was developed that can predict the scattering of irregular waves normally incident upon an array of vertical cylinders. It includes the pressure drop due to inertia resistance and the 
energy dissipation due to flow separation in front of and behind the cylinders. The nonlinear dissipation term due to flow separation was linearized by introducing a depth-averaged dissipation coefficient, which was obtained by equating the expectations of the depth-integrated powers of the linearized and nonlinear dissipation. For an array of circular cylinders for which the porosity varies along the gap, an ad hoc porosity was introduced to calculate the energy loss coefficient.

In order to examine the predictability of the developed model, laboratory experiments were made for the reflection and transmission of irregular waves from arrays of circular cylinders of various diameters and gap widths. Examining the frequency-averaged reflection and transmission coefficients, though the overall agreement between measurement and calculation is fairly good, as the gap width decreases, the model tends to over-predict the reflection coefficient and underpredict the transmission coefficient compared with the measurement. On the other hand, the model under-predicts the energy loss coefficients for small gap widths probably because the model neglects the evanescent waves near the cylinders, which may increase the energy loss through the gap between the cylinders.

The peaks of the measured spectra of the reflected and transmitted waves slightly shifted towards higher frequencies compared with that of the incident wave spectrum. Presently this seems to be attributed to the generation of shorter period waves due to the interference of the cylinders, but further study is needed to clarify this phenomenon.

Both measurement and calculation show that wave reflection and transmission become larger and smaller, respectively, as the wave steepness increases. Therefore the cylinder breakwater is more effective for steeper waves in protecting the harbor area while it is more effective for milder waves in reducing the wave reflection from the breakwater, which is a desirable feature of the cylinder breakwaters.

\section{Acknowledgements}

This work is partly a result of research sponsored by Korea Ocean Research and Development Institute under Project No. BSPE97628. KDS received support from Engineering Education and Research Foundation of Seoul National University under Contract No. 98-7026. The writers would like to thank SangYoung Son, who carried out some of the calculations in this paper.

\section{References}


Bennett, G. S., McIver, P. and Smallman, J. V. (1992). A mathematical model of a slotted wavescreen breakwater. Coastal Engrg. 18: 231-249.

Goda, Y. (1985). Random Seas and Design of Maritime Structures. Univ. of Tokyo Press, $323 \mathrm{pp}$.

Hagiwara, K. (1984). Analysis of upright structure for wave dissipation using integral equation. Proc. 19th Coastal Engrg. Conf., ASCE, pp. 2810-2826.

Hattori, M. (1972). Transmission of waves through perforated wall. Coastal Engrg. in Japan, 15: 69-79.

Hayashi, T., Hattori, M., Kano, T. and Shirai, M. (1966). Hydraulic research on the closely spaced pile breakwater. Proc. 10th Coastal Engrg. Conf., ASCE, pp. 873-884

Kakuno, S. and Liu, P. L.-F. (1993). Scattering of water waves by vertical cylinders. J. Wtrwy., Port, Coast. and Oc. Engrg., ASCE, 119: 302-322.

Kakuno, S. and Oda, K. (1986). Boundary value analysis on the interaction of cylinder arrays of arbitrary cross-section with train of uniform waves. $J$. Hydr., Coast. and Env. Engrg., Japan Soc. Civil Engrs., No. 369/II-5, pp. 213-222 (in Japanese).

Kim, B. H. (1998). Interactions of Waves, Seabed and Structures. Ph.D dissertation, Seoul Nat. Univ., 246 pp.

Kojima, H., Utsunomiya, M., Ijima, T., Yoshida, A. and Kihara, T. (1988). Analysis of hydraulic characteristics of permeable breakwaters to oblique incident waves. Proc. 35th Japanese Conf. Coastal Engrg., pp. 542-546 (in Japanese).

Kondo, H. (1979). Analysis of breakwaters having two porous walls. Proc. Coastal Structures'79, Vol. 2, pp. 962-977.

Lamb, H. (1932). Hydrodynamics. Cambridge Univ. Press, New York, 738pp.

Linton, C. M. and Evans, D. V. (1990). The interaction of waves with arrays of vertical circular cylinders. J. Fluid Mech., 215: 549-570.

Martin, P. A. and Dalrymple, R. A. (1988). Scattering of long waves by cylinders obstacles and gratings using matched asymptotic expansions. J. Fluid Mech., 188: $465-490$.

McBride, M. W., Smallman, J. V. and Allsop, N. W. H. (1994). Design of harbour entrances: breakwater design and vessel safety. Proc. Hydro-Port'94. Port and Harbour Research Institute, Yokosuka, Vol. 1, pp. 525-541.

Mei, C. C. (1983). The Applied Dynamics of Ocean Surface Waves. WileyInterscience, $740 \mathrm{pp}$.

Park, W. S., Oh, Y. M. and Chun, I. S. (1992). Separation technique of incident and reflected waves using least squares method. J. Korean Soc. Coast. and Oc. Engrs., 4: 139-145 (in Korean). 
Spring, B. H. and Monkmeyer, P. L. (1974). Interaction of plane waves with vertical cylinders. Proc. 14th Coastal Engrg. Conf., ASCE, pp. 1828-1845.

Srokosz, M. A. and Evans, D. V. (1979). A theory for wave power absorption by two independently oscillating bodies. J. Fluid Mech., 90: 337-362.

Twersky, V. (1962). On scattering waves by the infinite grating of circular cylinders. IRE Trans. Antennas and Propagation, 10: 737-765.

Uda, T., Omata, A. and Kawamura, T. (1990). An experimental study on wave dissipation and wave forces on the slit-type structures. Rep. Public Works Res. Inst., 2891, Japan Ministry of Construction (in Japanese). 
Table 1

Geometric parameters of the arrays of cylinders and corresponding coefficients

\begin{tabular}{rrrrrrrr}
\hline Case & $d(\mathrm{~cm})$ & $a(\mathrm{~cm})$ & $A(\mathrm{~cm})$ & $r_{0}$ & $\bar{r}$ & $\alpha$ & $C$ \\
\hline 1 & 11.5 & 2.08 & 7.83 & 0.266 & 0.359 & 6.20 & 12.9 \\
2 & 11.5 & 1.58 & 7.33 & 0.216 & 0.305 & 9.58 & 14.6 \\
3 & 11.5 & 1.08 & 6.83 & 0.158 & 0.241 & 17.52 & 18.0 \\
4 & 11.5 & 0.58 & 6.33 & 0.092 & 0.159 & 46.85 & 27.1 \\
5 & 11.5 & 0.33 & 6.08 & 0.054 & 0.107 & 113.50 & 42.0 \\
6 & 4.8 & 2.00 & 4.80 & 0.417 & 0.583 & 1.31 & 5.3 \\
7 & 4.8 & 1.00 & 3.40 & 0.294 & 0.387 & 4.96 & 5.1 \\
8 & 4.8 & 0.25 & 2.65 & 0.094 & 0.163 & 44.53 & 11.1 \\
\hline
\end{tabular}


Table 2

Frequency-averaged reflection and transmission coefficients of measured and calculated spectra

\begin{tabular}{|c|c|c|c|c|c|c|c|c|c|c|c|c|c|}
\hline \multirow[t]{2}{*}{ Case } & \multirow[t]{2}{*}{$T_{s} \quad(\mathrm{~s})$} & \multicolumn{6}{|c|}{$H_{s} \approx 5 \mathrm{~cm}$} & \multicolumn{6}{|c|}{$H_{s} \approx 10 \mathrm{~cm}$} \\
\hline & & $K_{R c}$ & $K_{R m}$ & Error & $K_{T c}$ & $K_{T m}$ & Error & $K_{R c}$ & $K_{R m}$ & Error & $K_{T c}$ & $K_{T m}$ & Error \\
\hline \multirow[t]{6}{*}{1} & 1.0 & .499 & .516 & -3.4 & .727 & .713 & 1.9 & & & & & & \\
\hline & 1.2 & .433 & .460 & -6.2 & .764 & .769 & -0.7 & & & & & & \\
\hline & 1.4 & .373 & .416 & -11.5 & .794 & .798 & -0.5 & .381 & .410 & -7.6 & .720 & .769 & -6.8 \\
\hline & 1.6 & .340 & .386 & -13.5 & .814 & .808 & 0.7 & .362 & .364 & -0.3 & .737 & .766 & -3.9 \\
\hline & 1.8 & .322 & .367 & -14.0 & .830 & .813 & 2.0 & .342 & .351 & -2.6 & .756 & .768 & -1.6 \\
\hline & 2.0 & .312 & .364 & -16.7 & .844 & .831 & 1.5 & .331 & .344 & -3.9 & .772 & .761 & 1.4 \\
\hline \multirow[t]{6}{*}{2} & 1.0 & .528 & .524 & 0.8 & .672 & .674 & -0.3 & & & & & & \\
\hline & 1.2 & .463 & .479 & -3.5 & .711 & .731 & -2.8 & & & & & & \\
\hline & 1.4 & .410 & .437 & -6.6 & .738 & .757 & -2.6 & .427 & .411 & 3.7 & .655 & .721 & -10.1 \\
\hline & 1.6 & .373 & .398 & -6.7 & .762 & .769 & -0.9 & .408 & .376 & 7.8 & .672 & .726 & -8.0 \\
\hline & 1.8 & .356 & .376 & -5.6 & .780 & .774 & 0.8 & .387 & .363 & 6.2 & .694 & .725 & -4.5 \\
\hline & 2.0 & .343 & .369 & -7.6 & .797 & .780 & 2.1 & .372 & .353 & 5.1 & .713 & .721 & -1.1 \\
\hline \multirow[t]{6}{*}{3} & 1.0 & .584 & .546 & 6.3 & .584 & .593 & -1.5 & & & & & & \\
\hline & 1.2 & .522 & .498 & 4.6 & .624 & .641 & -2.7 & & & & & & \\
\hline & 1.4 & .470 & .451 & 4.0 & .652 & .674 & -3.4 & .503 & .451 & 10.3 & .560 & .637 & -13.8 \\
\hline & 1.6 & .435 & .423 & 2.8 & .677 & .692 & -2.2 & .483 & .415 & 14.1 & .577 & .636 & -10.2 \\
\hline & 1.8 & .417 & .408 & 2.2 & .697 & .700 & -0.4 & .465 & .398 & 14.4 & .597 & .630 & -5.5 \\
\hline & 2.0 & .405 & .399 & 1.5 & .715 & .701 & 2.0 & .448 & .403 & 10.0 & .617 & .626 & -1.5 \\
\hline \multirow[t]{6}{*}{4} & 1.0 & .688 & .602 & 12.5 & .432 & .473 & -9.5 & & & & & & \\
\hline & 1.2 & .634 & .558 & 12.0 & .471 & .505 & -7.2 & & & & & & \\
\hline & 1.4 & .589 & .521 & 11.5 & .498 & .532 & -6.8 & .635 & .539 & 15.1 & .406 & .480 & -18.2 \\
\hline & 1.6 & .561 & .498 & 11.2 & .516 & .539 & -4.5 & .622 & .511 & 17.8 & .415 & .481 & -15.9 \\
\hline & 1.8 & .543 & .482 & 11.2 & .536 & .542 & -1.1 & .605 & .502 & 17.0 & .433 & .474 & -9.5 \\
\hline & 2.0 & .530 & .482 & 9.1 & .555 & .545 & 1.8 & .589 & .510 & 13.4 & .452 & .471 & -4.2 \\
\hline \multirow[t]{6}{*}{5} & 1.0 & .777 & .638 & 17.9 & .312 & .378 & -21.2 & & & & & & \\
\hline & 1.2 & .735 & .616 & 16.2 & .342 & .397 & -16.1 & & & & & & \\
\hline & 1.4 & .700 & .584 & 16.6 & .364 & .405 & -11.3 & .741 & .625 & 15.7 & .287 & .359 & -25.1 \\
\hline & 1.6 & .678 & .578 & 14.7 & .377 & .409 & -8.5 & .733 & .609 & 16.9 & .293 & .355 & -21.2 \\
\hline & 1.8 & .664 & .567 & 14.6 & .393 & .398 & -1.3 & .720 & .609 & 15.4 & .306 & .347 & -13.4 \\
\hline & 2.0 & .652 & .576 & 11.7 & .410 & .401 & 2.2 & .708 & .617 & 12.9 & .320 & .343 & -7.2 \\
\hline \multirow[t]{6}{*}{6} & 1.0 & .280 & .236 & 15.7 & .910 & .900 & 1.1 & & & & & & \\
\hline & 1.2 & .238 & .181 & 23.9 & .925 & .916 & 1.0 & & & & & & \\
\hline & 1.4 & .193 & .156 & 19.2 & .939 & .916 & 2.4 & .187 & .181 & 3.2 & .907 & .918 & -1.0 \\
\hline & 1.6 & .165 & .144 & 12.7 & .948 & .917 & 3.3 & .174 & .166 & 4.6 & .915 & .899 & 1.7 \\
\hline & 1.8 & .156 & .141 & 9.6 & .953 & .915 & 4.0 & .163 & .167 & -2.5 & .923 & .890 & 3.6 \\
\hline & 2.0 & .146 & .139 & 4.8 & .959 & .909 & 5.2 & .155 & .164 & -5.8 & .931 & .878 & 5.7 \\
\hline \multirow[t]{6}{*}{7} & 1.0 & .282 & .273 & 3.2 & .815 & .843 & -3.4 & & & & & & \\
\hline & 1.2 & .247 & .213 & 13.8 & .834 & .865 & -3.7 & & & & & & \\
\hline & 1.4 & .213 & .186 & 12.7 & .851 & .869 & -2.1 & .260 & .226 & 13.1 & .772 & .845 & -9.5 \\
\hline & 1.6 & .188 & .173 & 8.0 & .865 & .877 & -1.4 & .243 & .197 & 18.9 & .787 & .831 & -5.6 \\
\hline & 1.8 & .175 & .166 & 5.1 & .877 & .872 & 0.6 & .227 & .201 & 11.5 & .803 & .830 & -3.4 \\
\hline & 2.0 & .168 & .167 & 0.6 & .888 & .873 & 1.7 & .213 & .205 & 3.8 & .819 & .815 & 0.5 \\
\hline \multirow[t]{6}{*}{8} & 1.0 & .558 & .470 & 15.8 & .479 & .530 & -10.6 & & & & & & \\
\hline & 1.2 & .528 & .436 & 17.4 & .507 & .548 & -8.1 & & & & & & \\
\hline & 1.4 & .499 & .410 & 17.8 & .527 & .560 & -6.3 & .592 & .482 & 18.6 & .419 & .499 & -19.1 \\
\hline & 1.6 & .480 & .400 & 16.7 & .541 & .567 & -4.8 & .581 & .473 & 18.6 & .429 & .495 & -15.4 \\
\hline & 1.8 & .461 & .399 & 13.4 & .559 & .559 & 0.0 & .563 & .466 & 17.2 & .447 & .482 & -7.8 \\
\hline & 2.0 & .441 & .406 & 7.9 & .581 & .565 & 2.8 & .543 & .479 & 11.8 & .467 & .472 & -1.1 \\
\hline
\end{tabular}




\section{Caption of Figures}

1. Schematic diagram and coordinate system for calculation of wave scattering by vertical cylinders.

2. Arrangement of wave gauges for measuring wave reflection and transmission.

3. Comparison of frequency-averaged reflection and transmission coefficients between experiment and theory: $=$ reflection; $O=$ transmission.

4. Values of $K_{R c} / K_{R m}$ as function of porosity $r_{0}$ : (a) $H_{s} \approx 5 \mathrm{~cm}$; (b) $H_{s} \approx$ $10 \mathrm{~cm}$.

5. Values of $K_{T c} / K_{T m}$ as function of porosity $r_{0}$ : (a) $H_{s} \approx 5 \mathrm{~cm}$; (b) $H_{s} \approx 10$ $\mathrm{cm}$.

6. Values of $K_{L c} / K_{L m}$ as function of porosity $r_{0}$ : (a) $H_{s} \approx 5 \mathrm{~cm}$; (b) $H_{s} \approx$ $10 \mathrm{~cm}$.

7. Reflection coefficients of steep waves against those of mild waves as function of porosity $r_{0}$ : (a) measurement; (b) calculation.

8. Transmission coefficients of steep waves against those of mild waves as function of porosity $r_{0}$ : (a) measurement; (b) calculation.

9. Measured and calculated spectra of incident, reflected and transmitted waves for Case 1 with $T_{s} \approx 1.6 \mathrm{~s}$ and $H_{s} \approx 5 \mathrm{~cm}$ : thick lines = measurement, thin lines = calculation.

10. Same as Fig. 9, but for Case 3 with $T_{s} \approx 1.6 \mathrm{~s}$ and $H_{s} \approx 5 \mathrm{~cm}$.

11. Same as Fig. 9, but for Case 5 with $T_{s} \approx 1.6 \mathrm{~s}$ and $H_{s} \approx 5 \mathrm{~cm}$.

12. Same as Fig. 9, but for Case 3 with $T_{s} \approx 1.6 \mathrm{~s}$ and $H_{s} \approx 10 \mathrm{~cm}$.

13. Same as Fig. 9, but for Case 3 with $T_{s} \approx 1.2 \mathrm{~s}$ and $H_{s} \approx 5 \mathrm{~cm}$.

14. Same as Fig. 9, but for Case 3 with $T_{s} \approx 2.0 \mathrm{~s}$ and $H_{s} \approx 5 \mathrm{~cm}$.

15. Same as Fig. 9, but for Case 2 with $T_{s} \approx 1.0 \mathrm{~s}$ and $H_{s} \approx 5 \mathrm{~cm}$.

16. Same as Fig. 9, but for Case 5 with $T_{s} \approx 1.4 \mathrm{~s}$ and $H_{s} \approx 10 \mathrm{~cm}$. 
Fig. 1
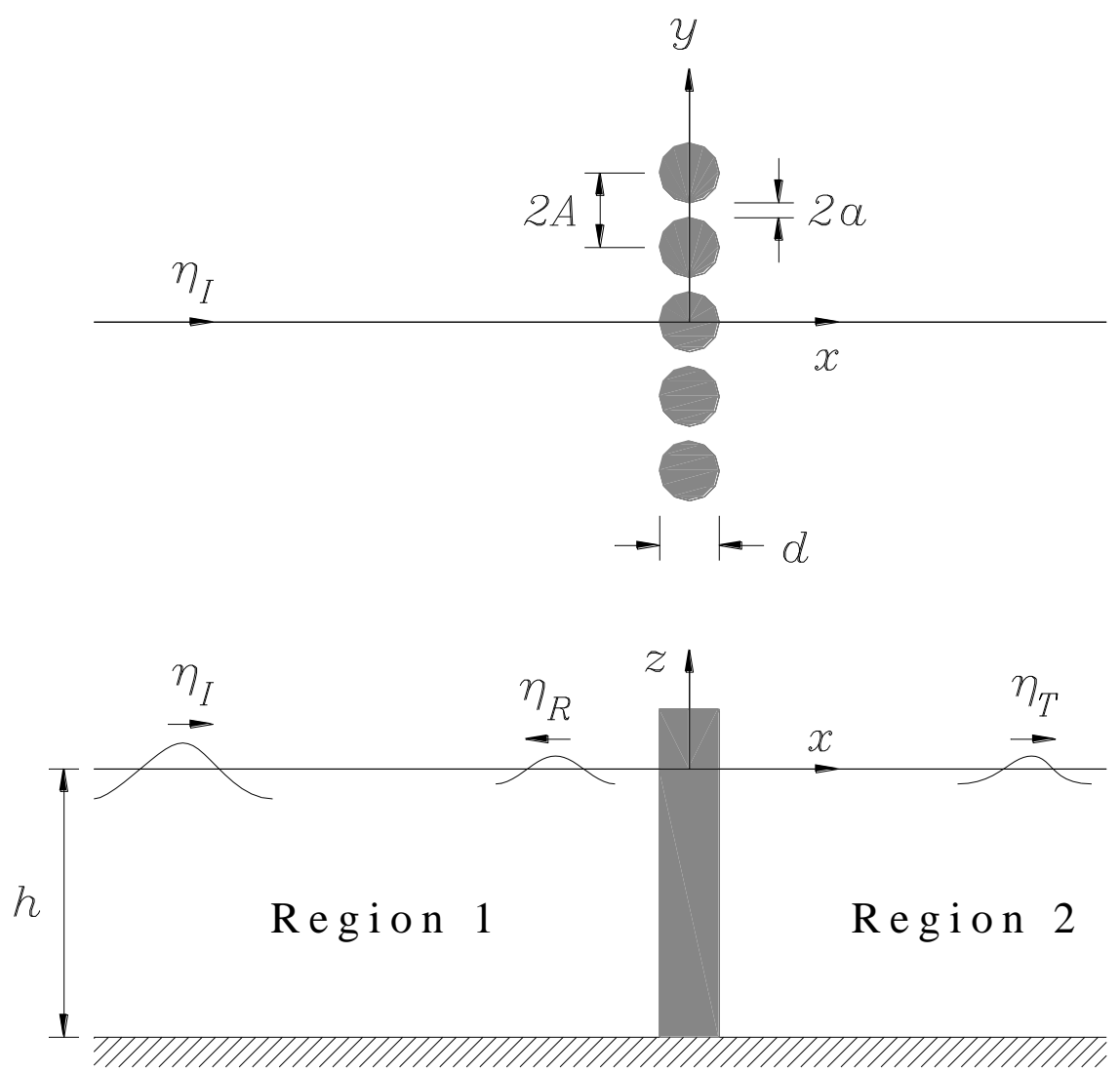
Fig. 2

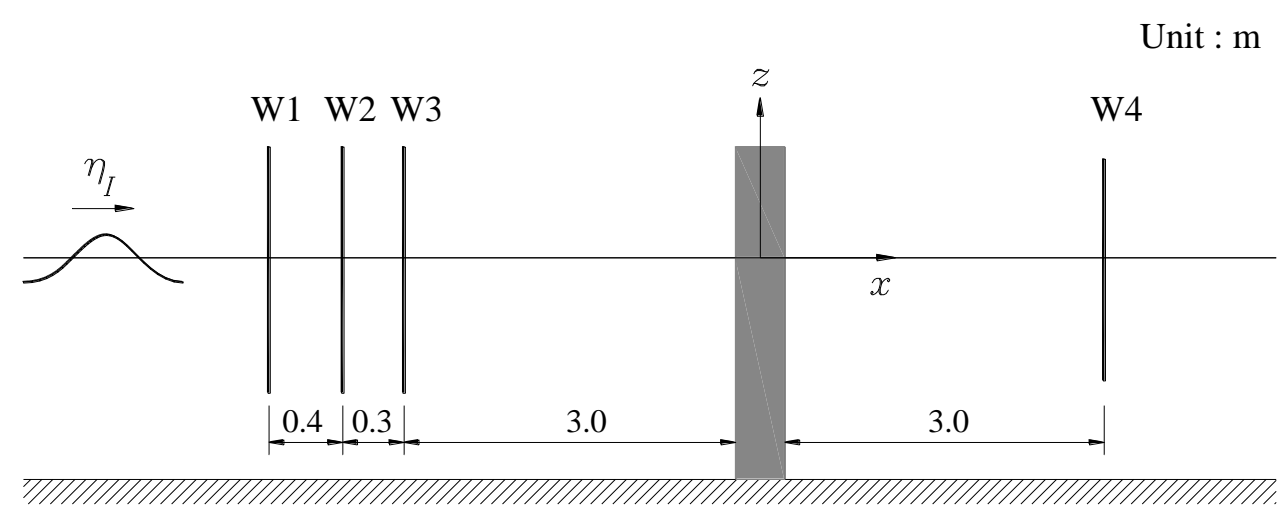


Fig. 3

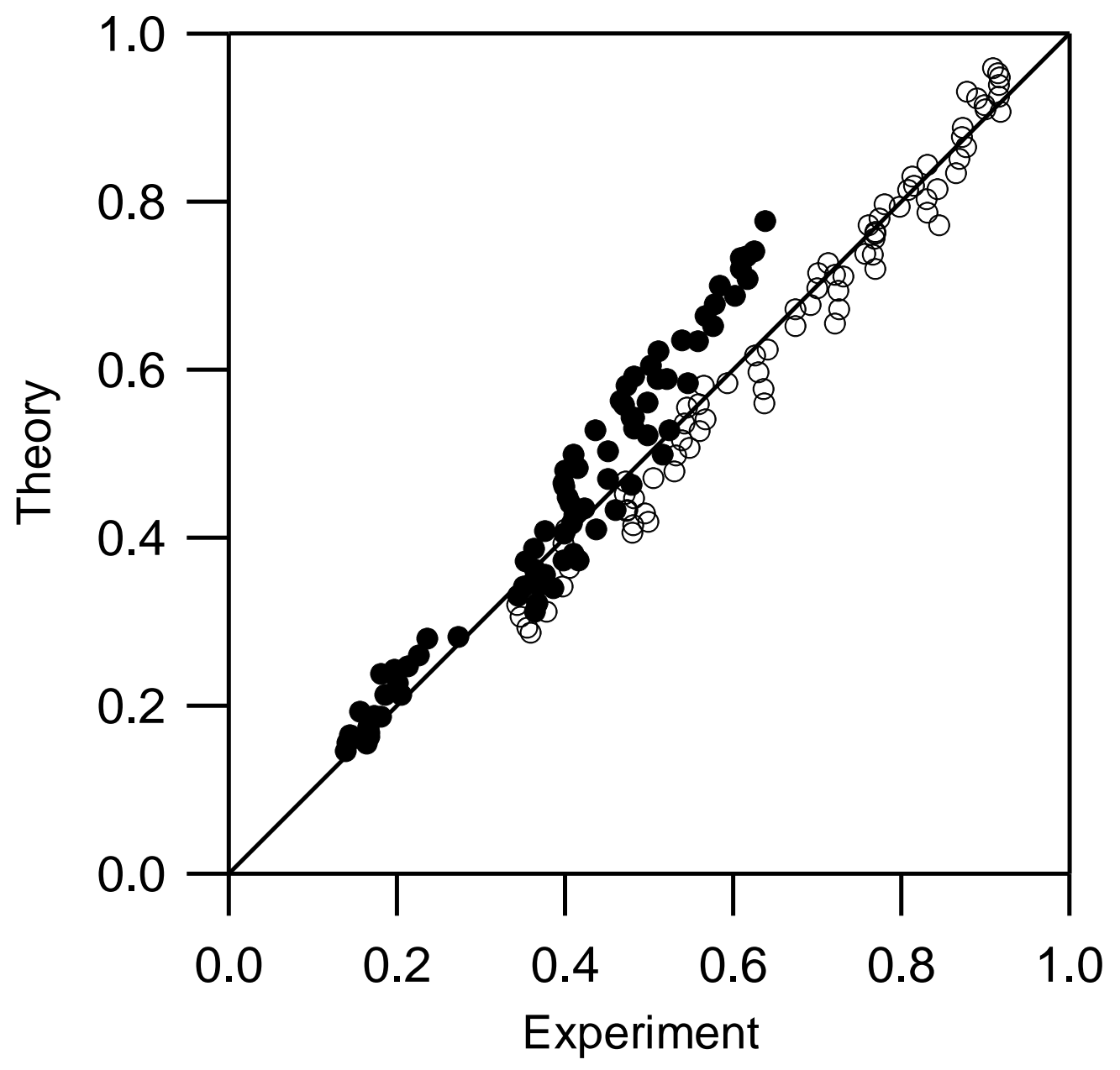


Fig. 4 (a)

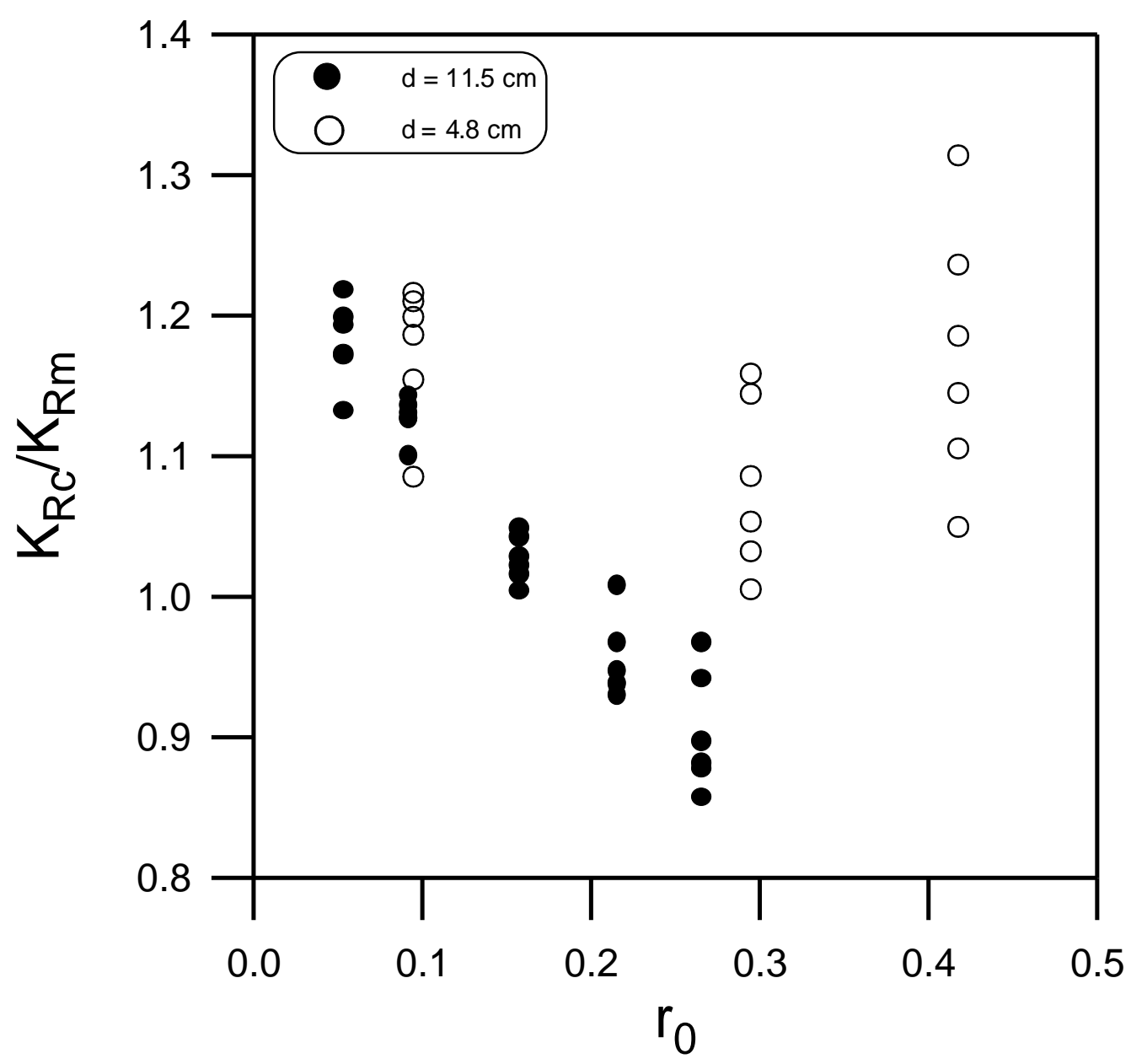


Fig. 4 (b)

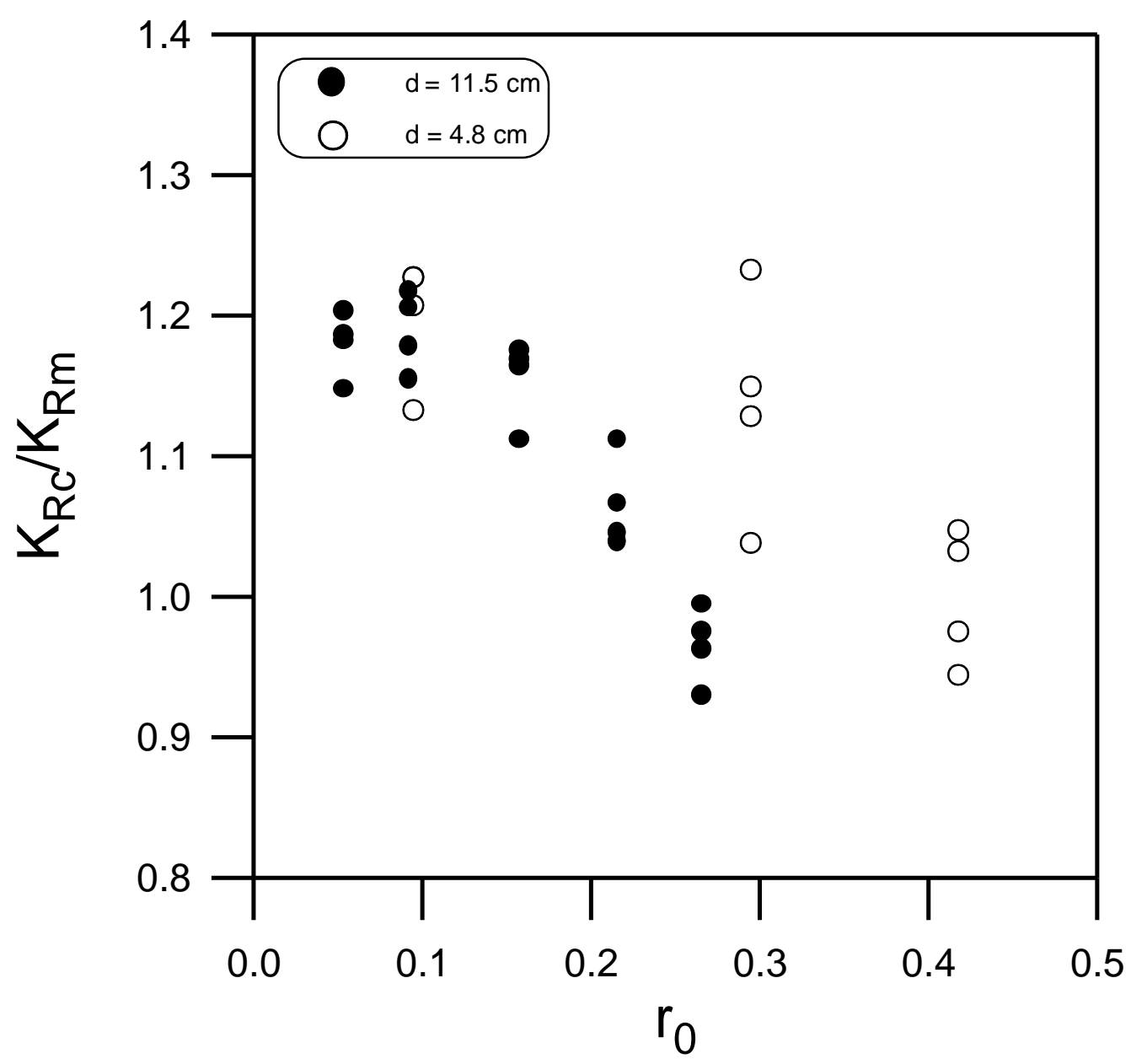


Fig. 5 (a)

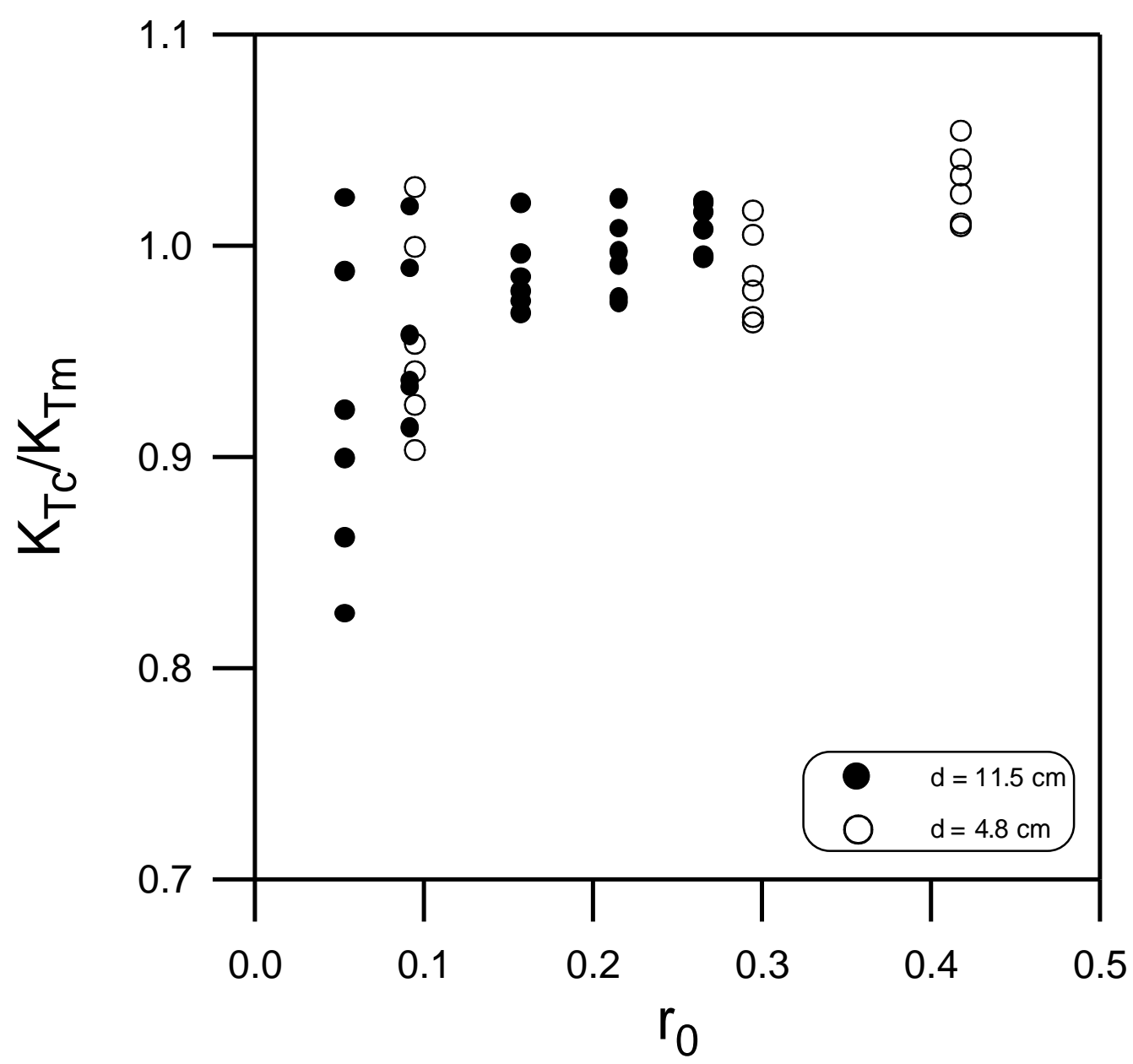


Fig. 5 (b)

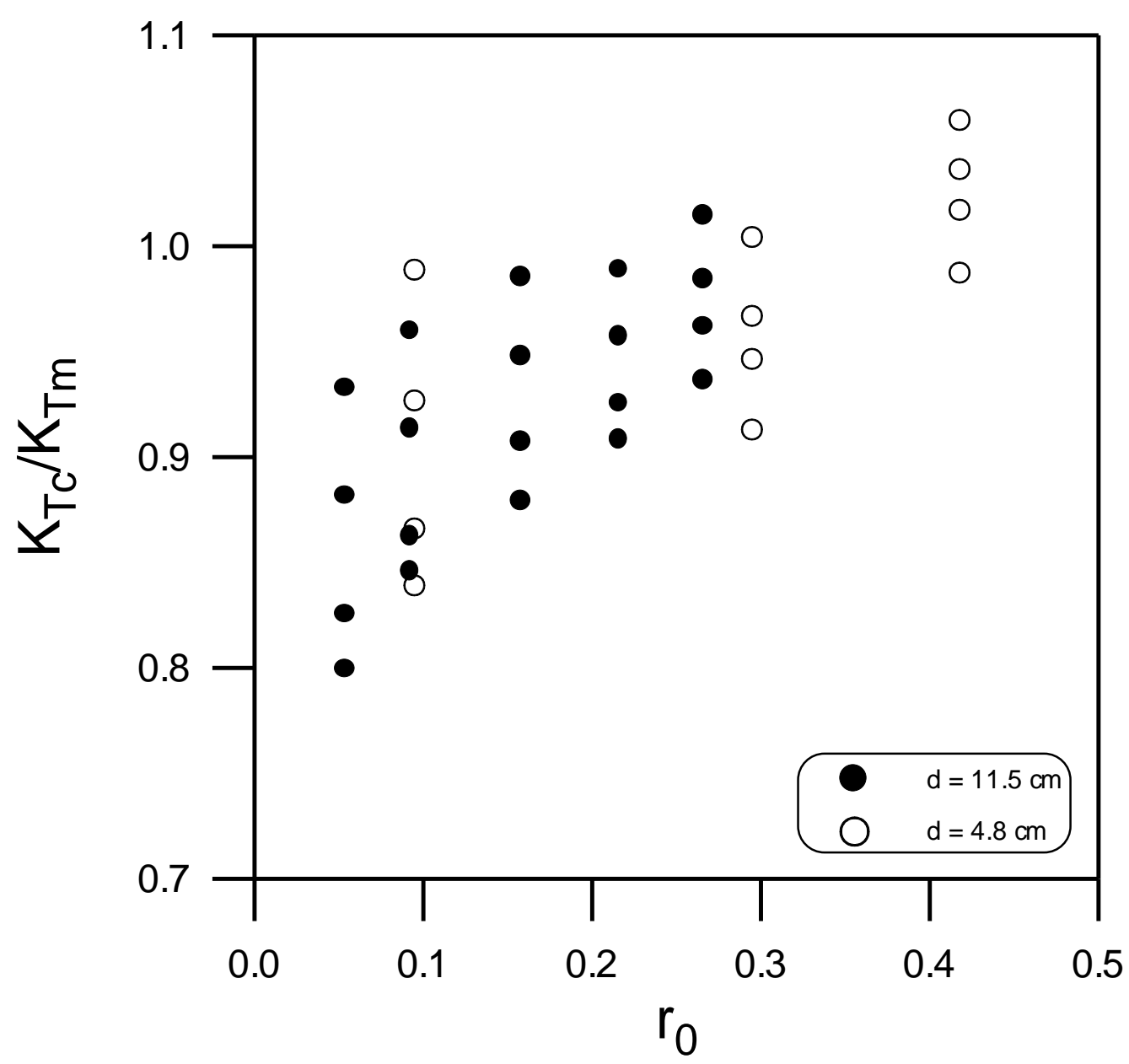


Fig. 6 (a)

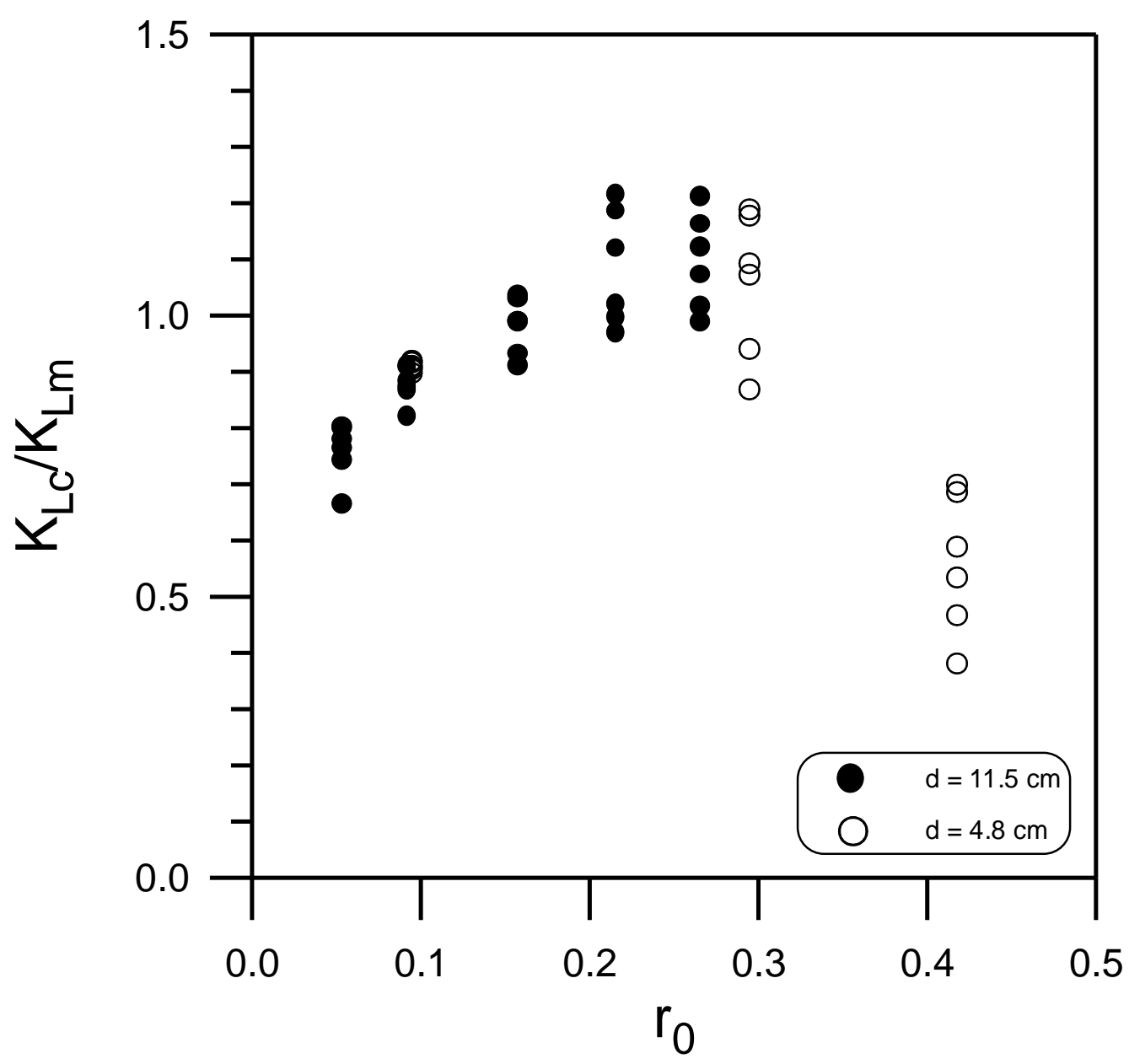


Fig. 6 (b)

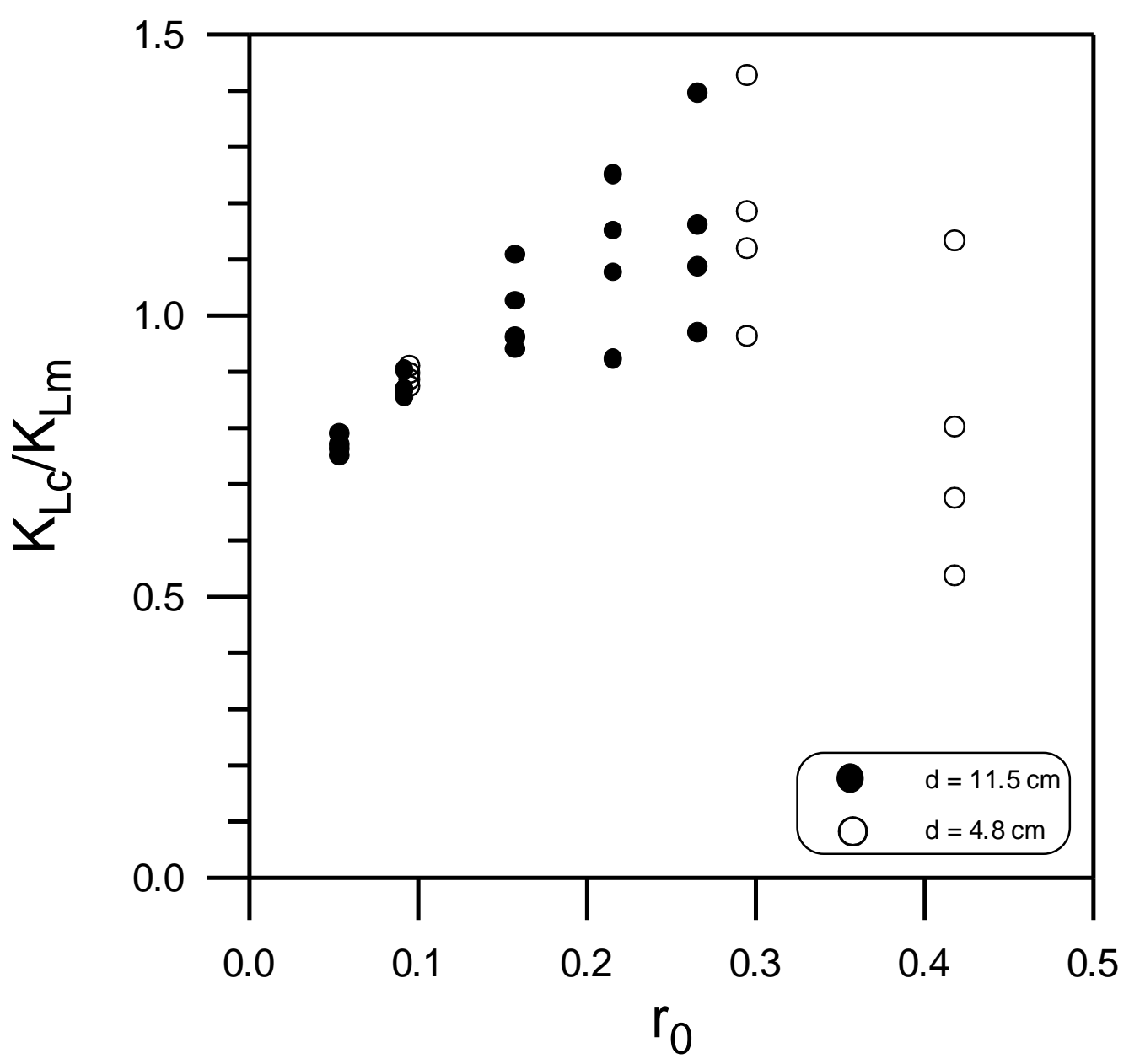


Fig. 7 (a)

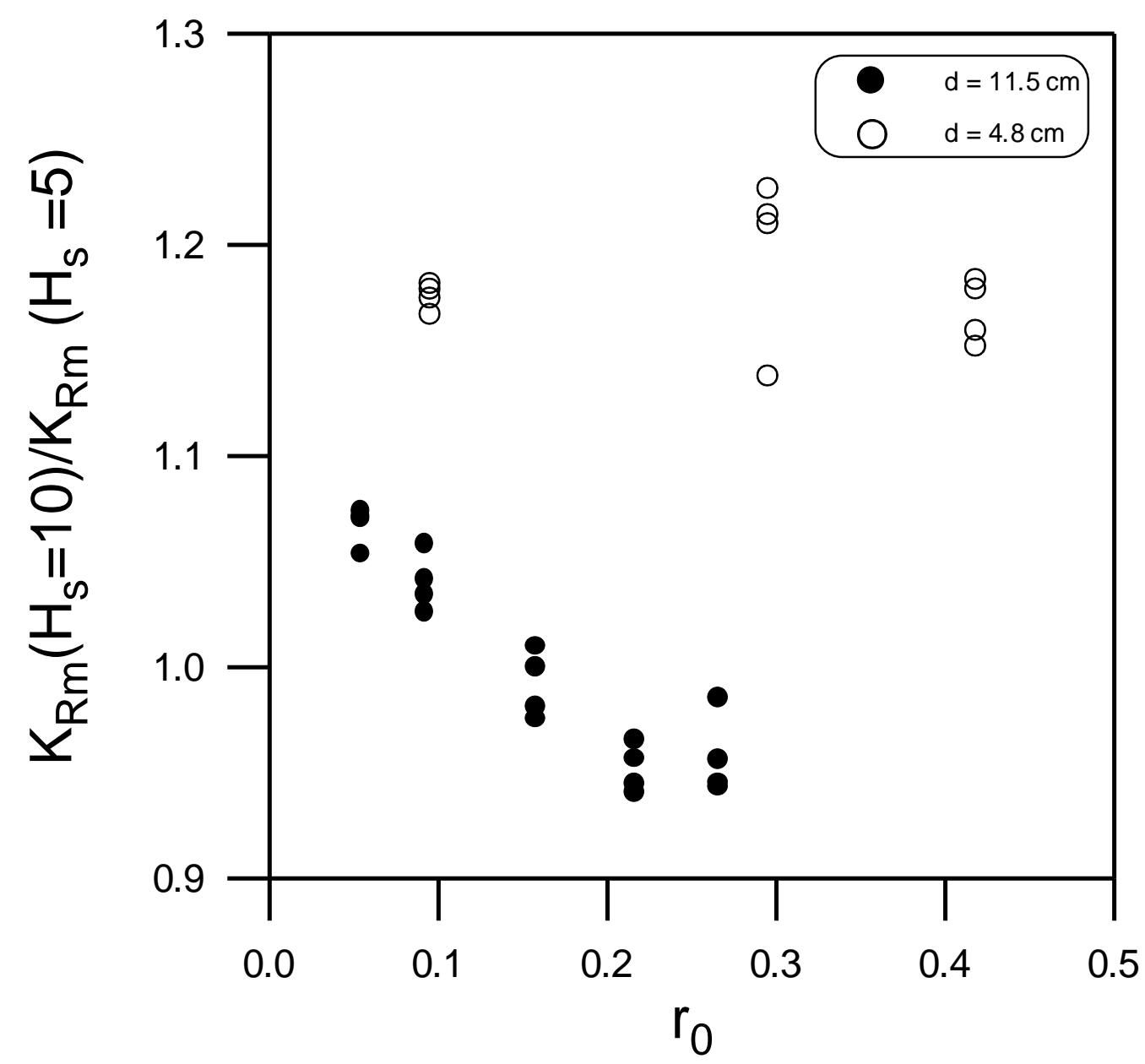


Fig. 7 (b)

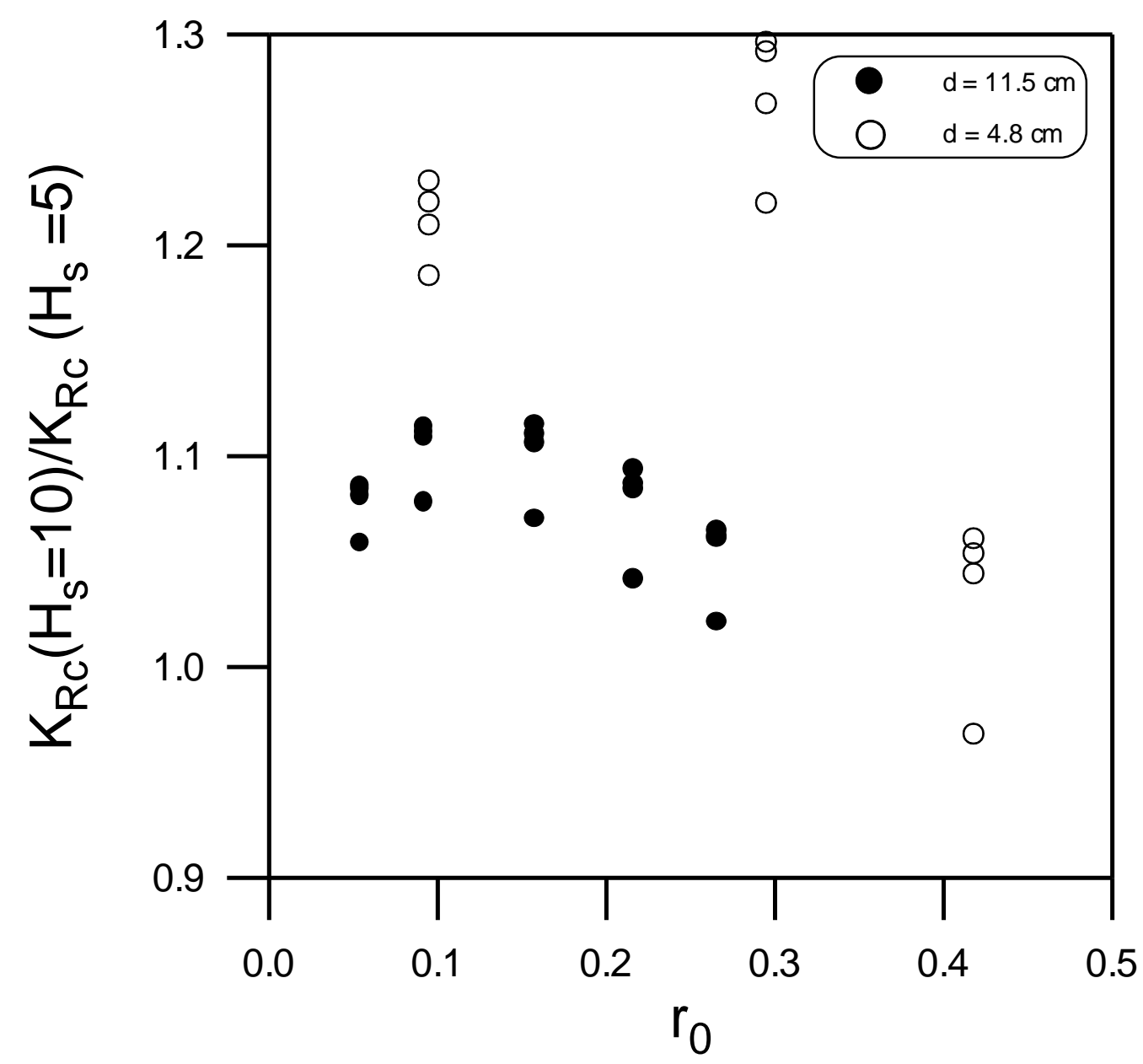


Fig. 8 (a)

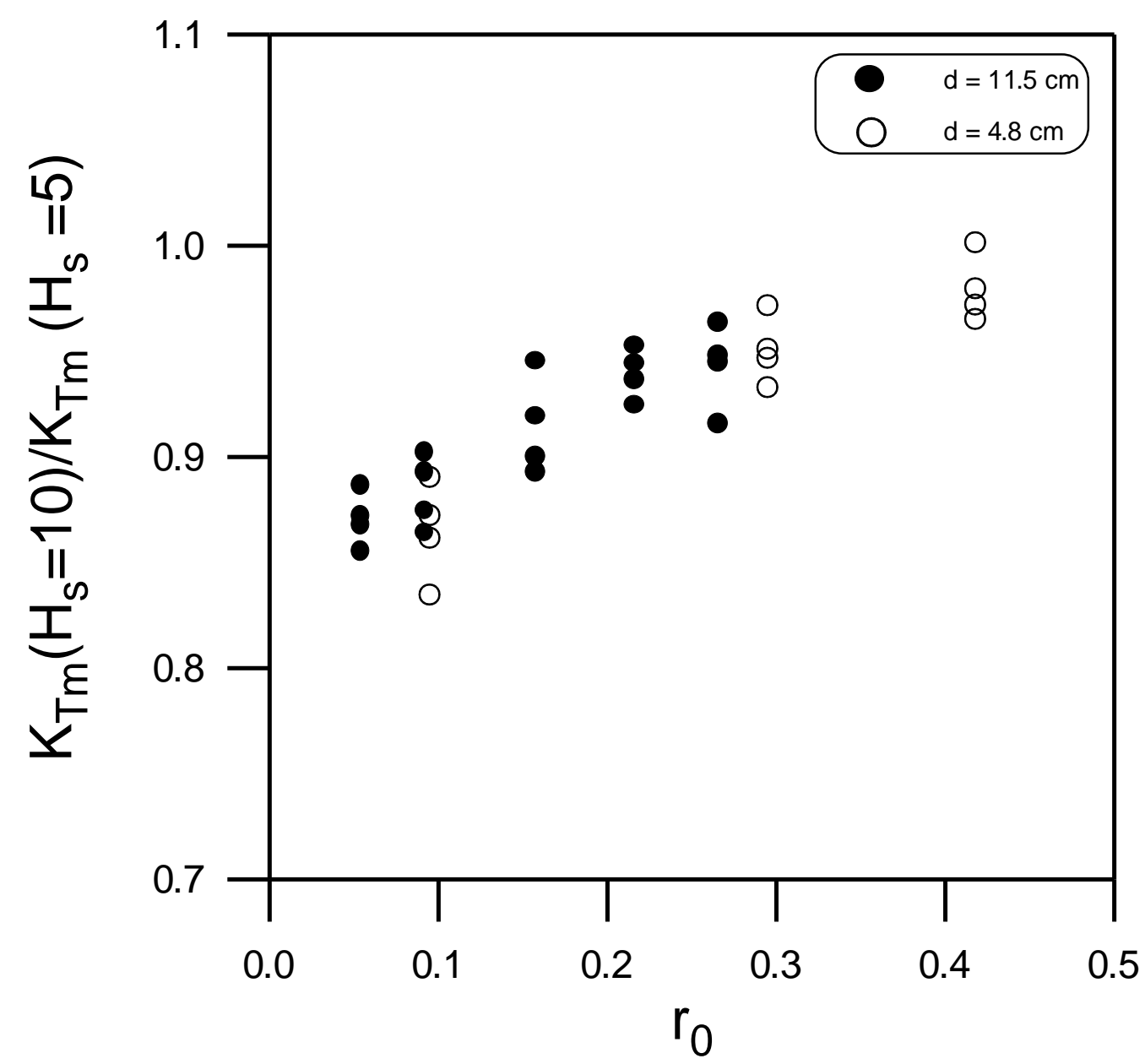


Fig. 8 (b)

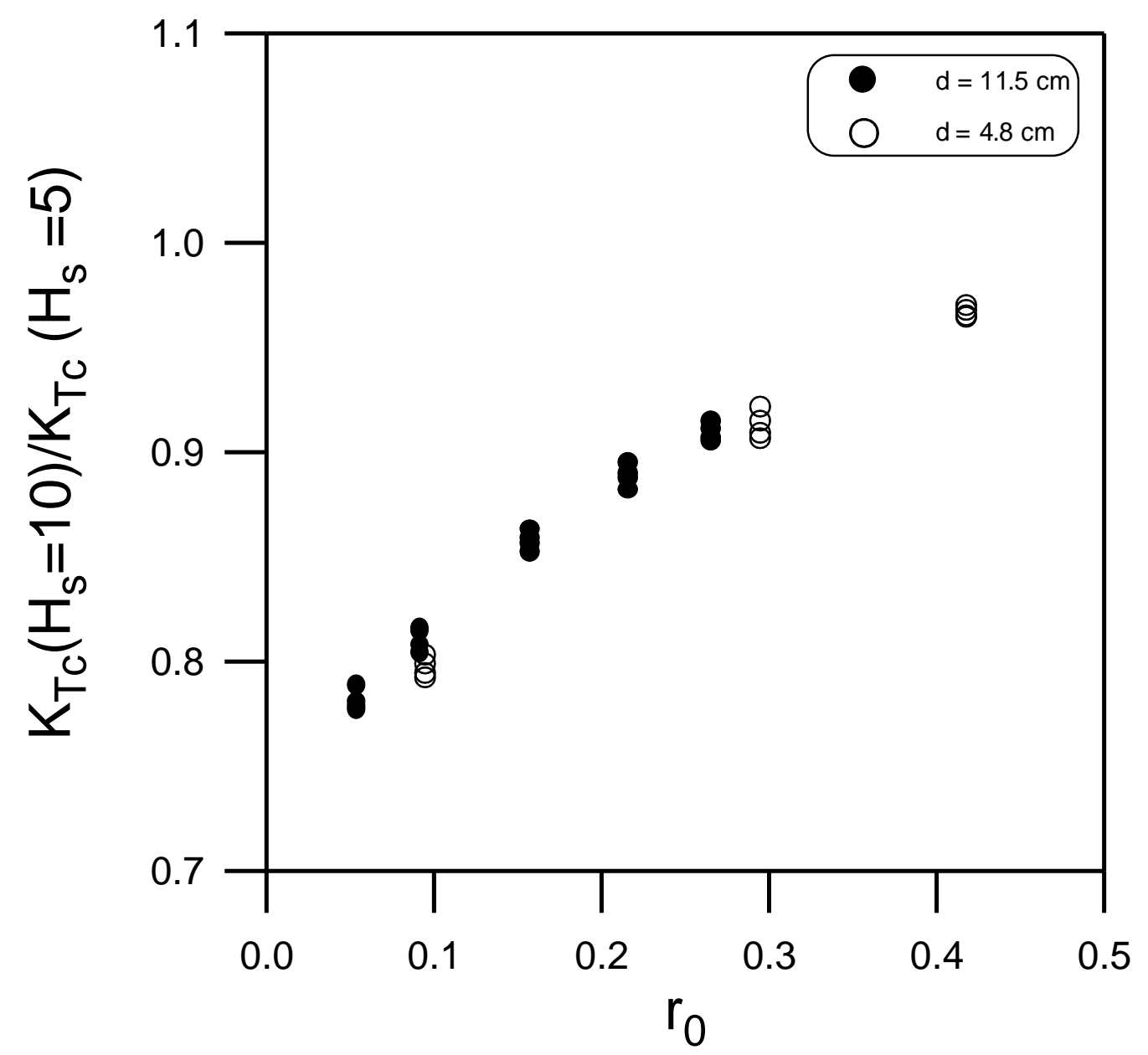


Fig. 9

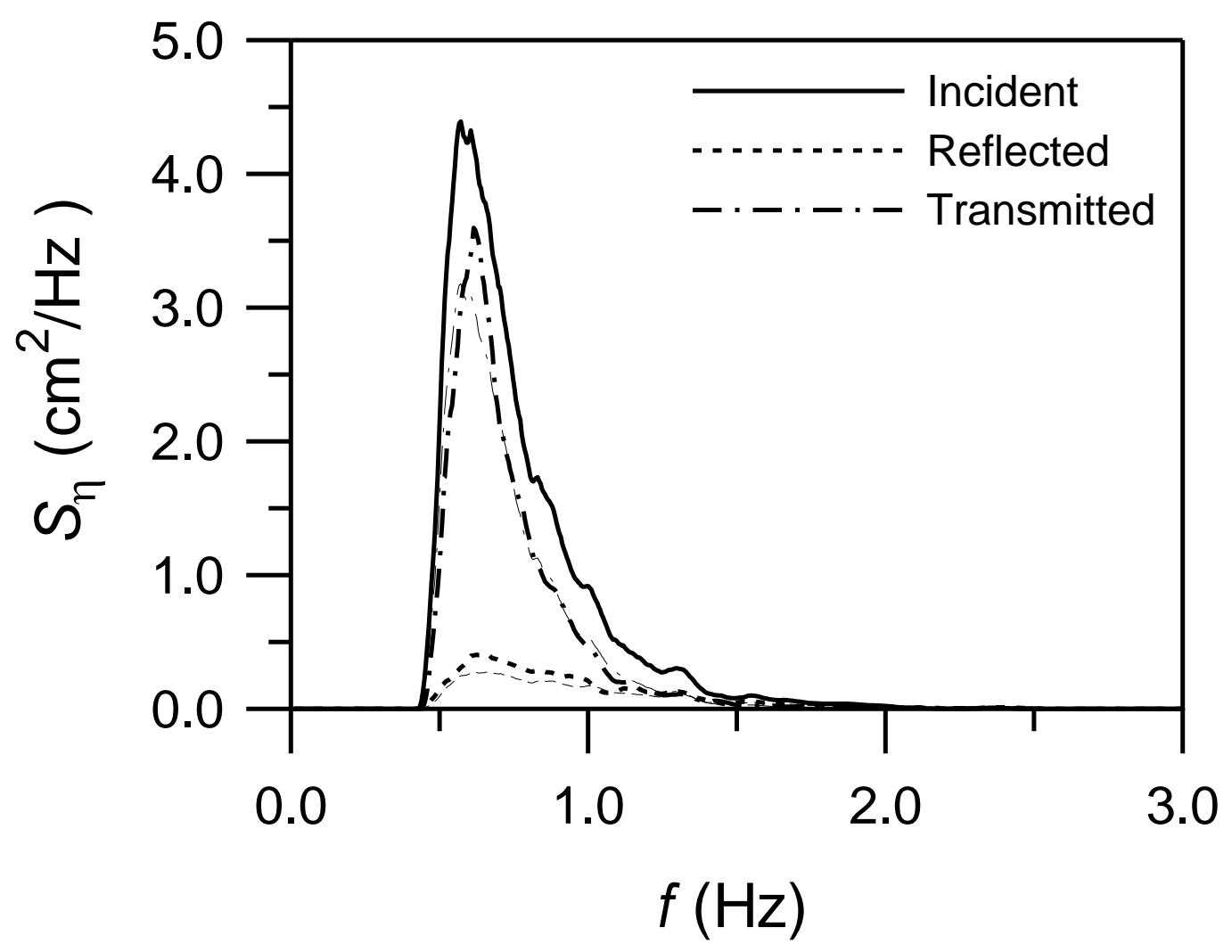


Fig. 10

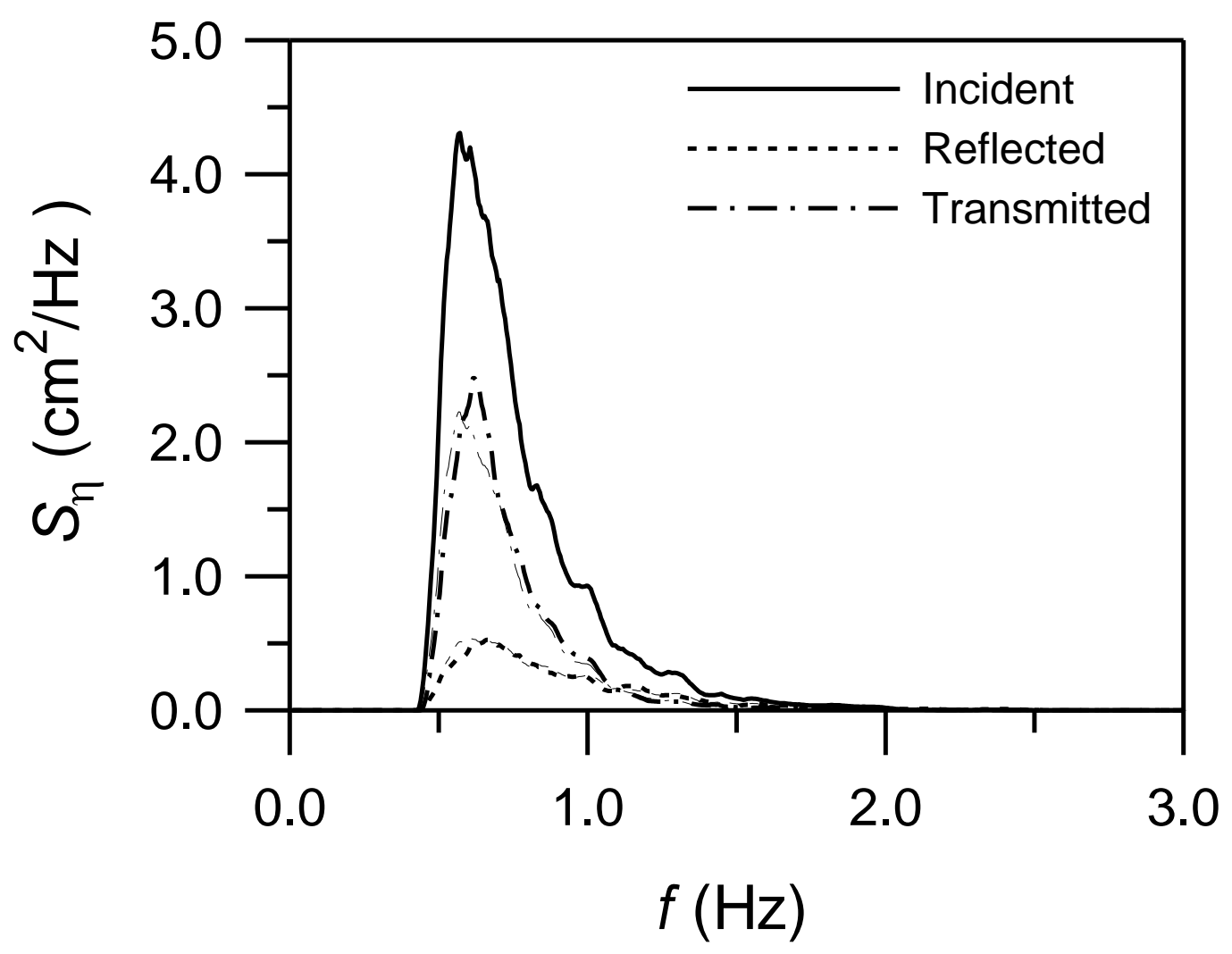


Fig. 11

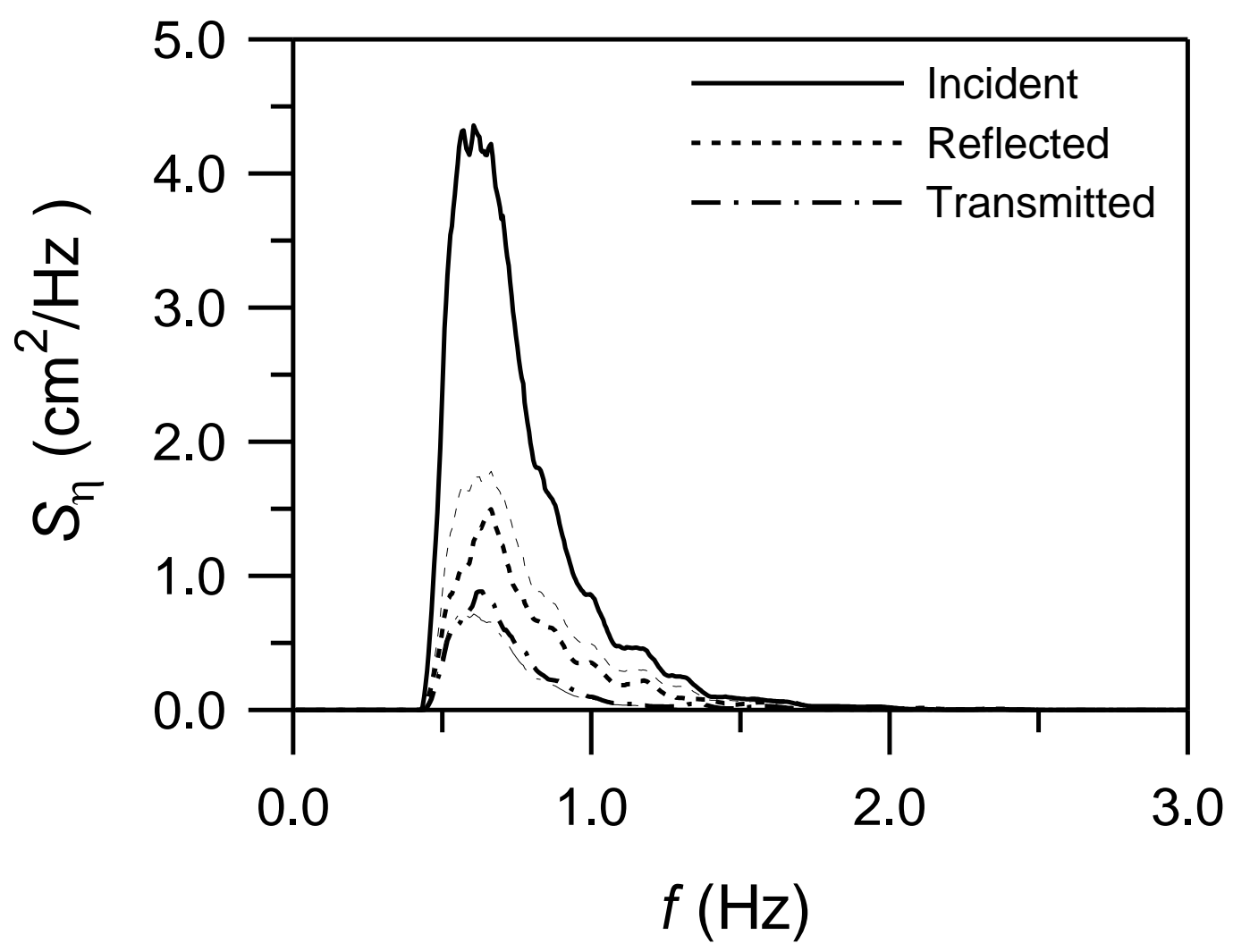


Fig. 12

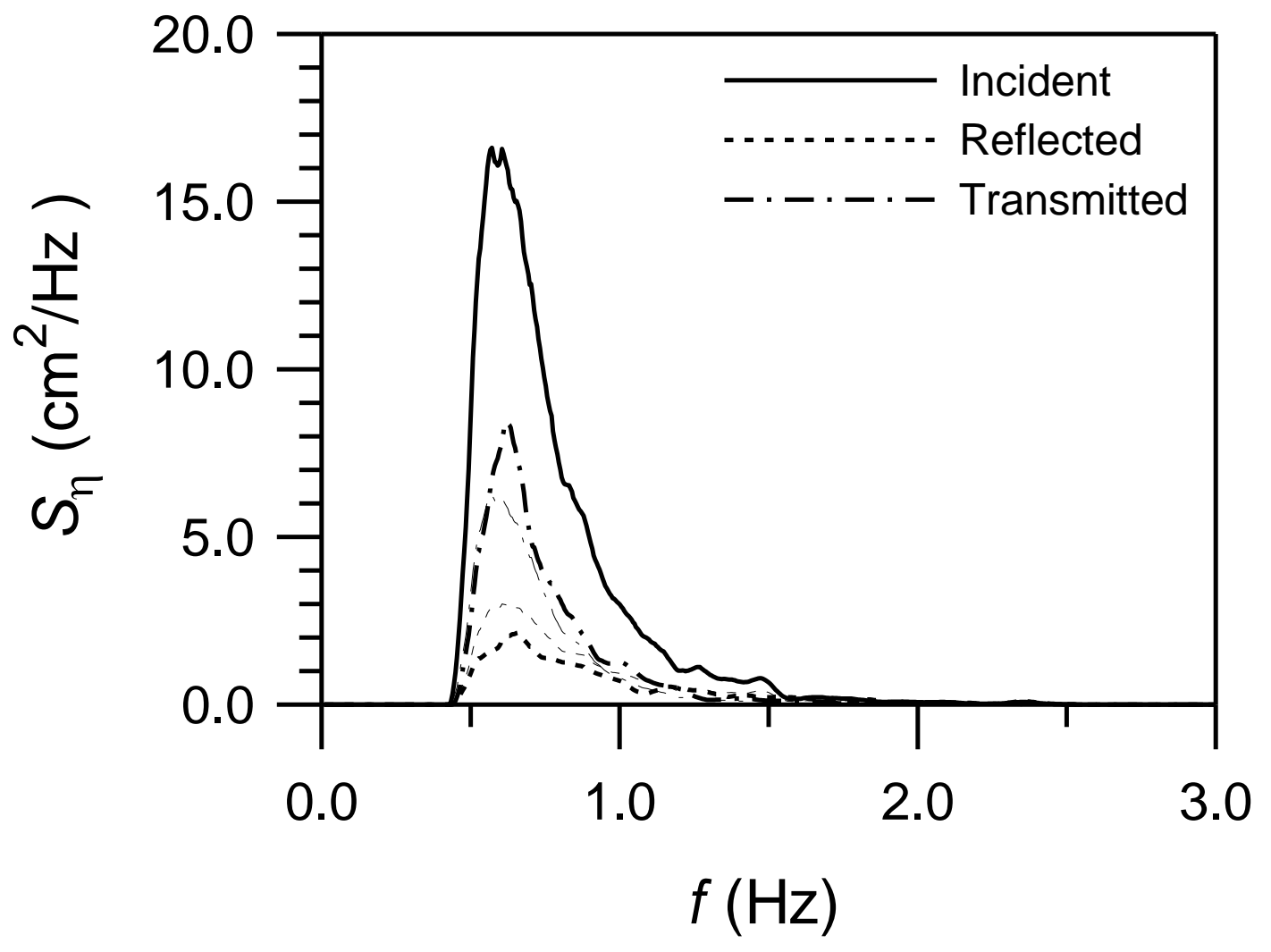


Fig. 13

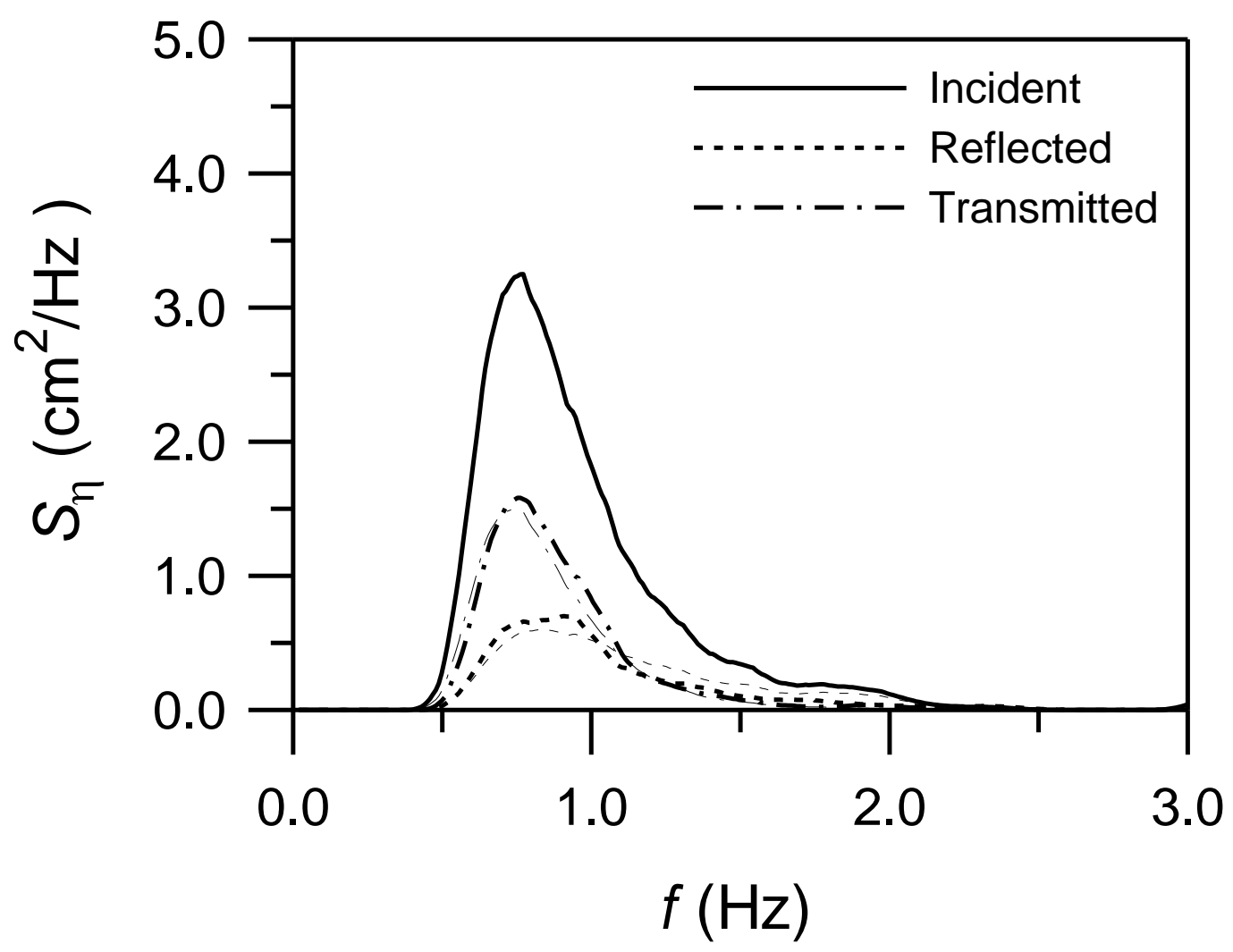


Fig. 14

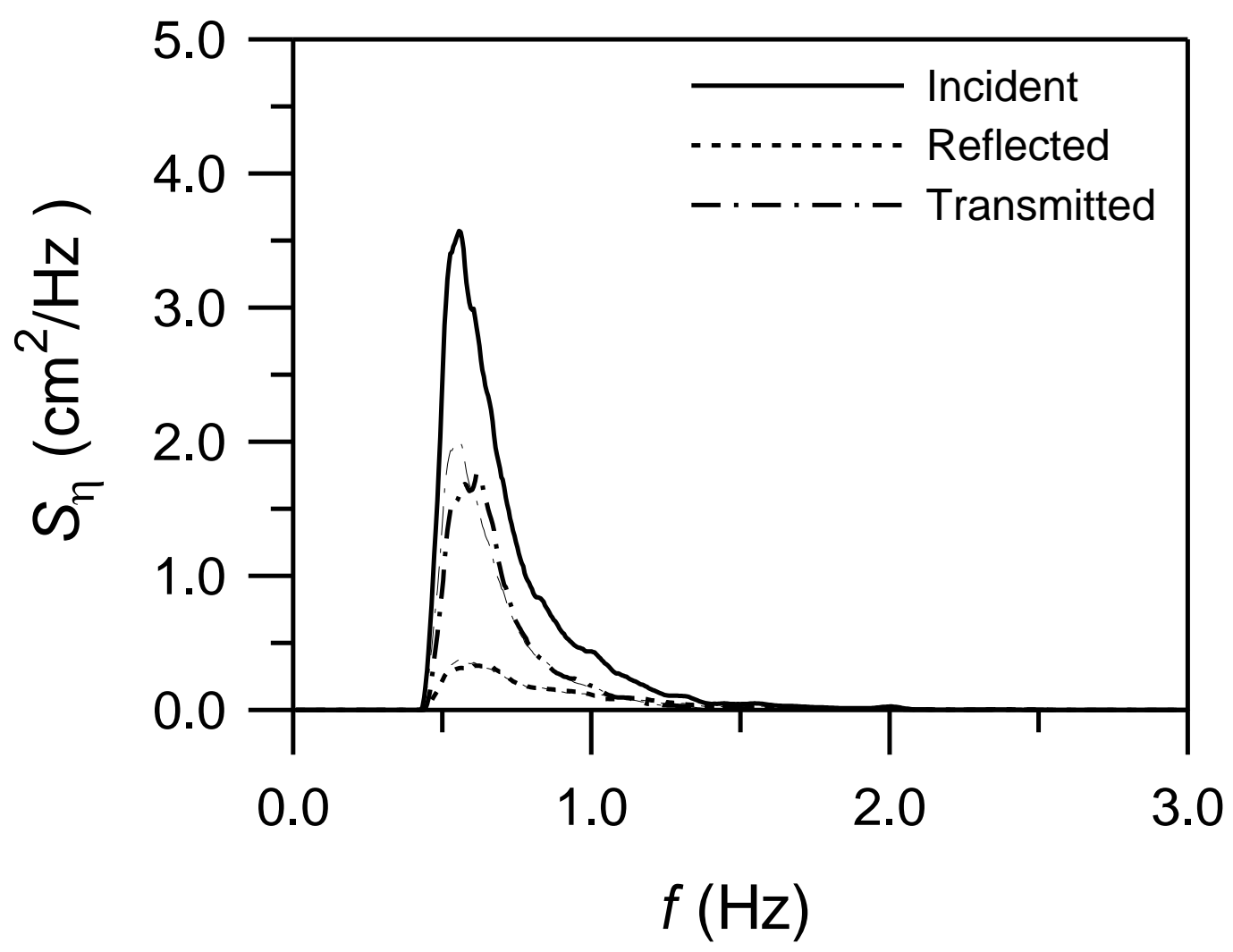


Fig. 15

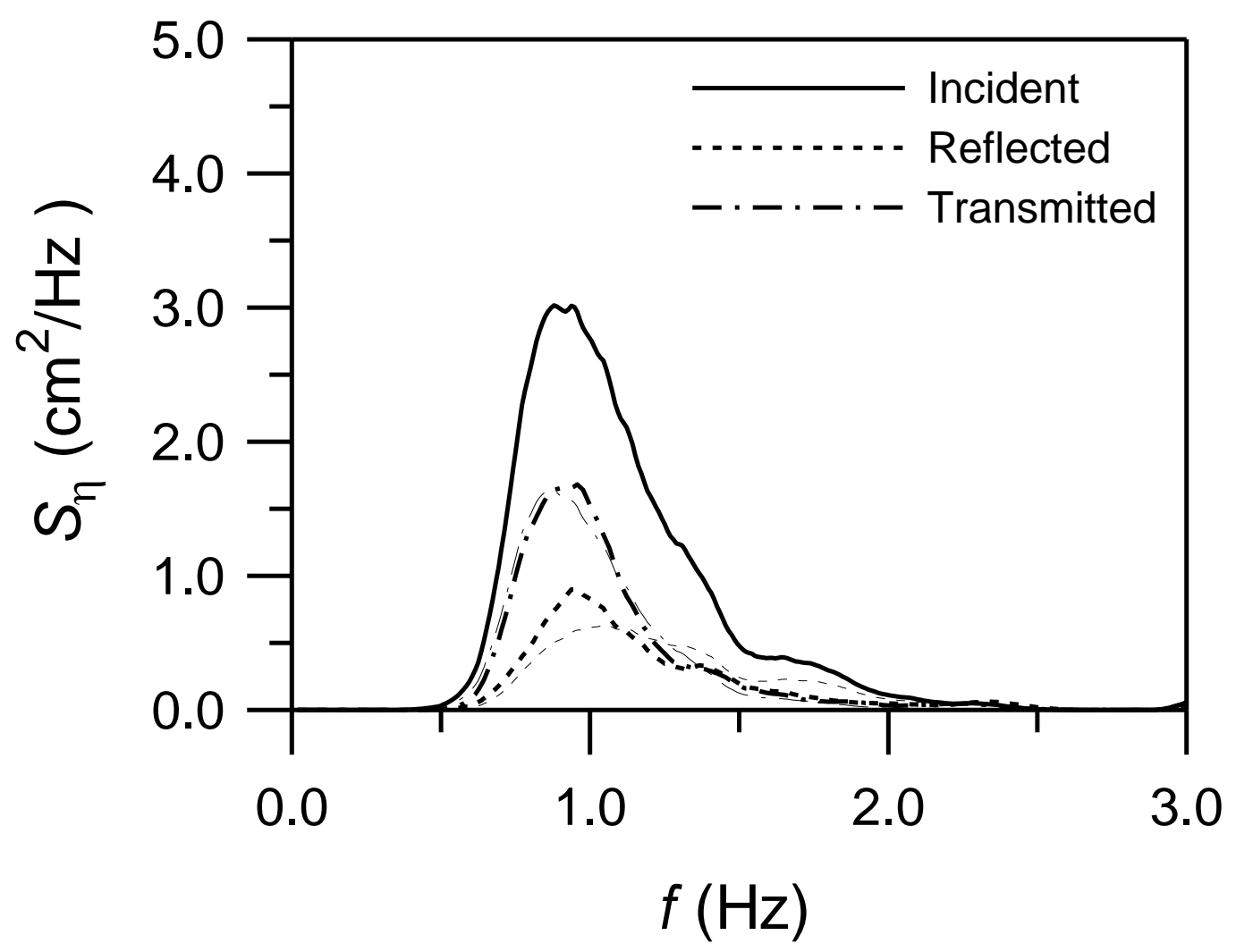


Fig. 16

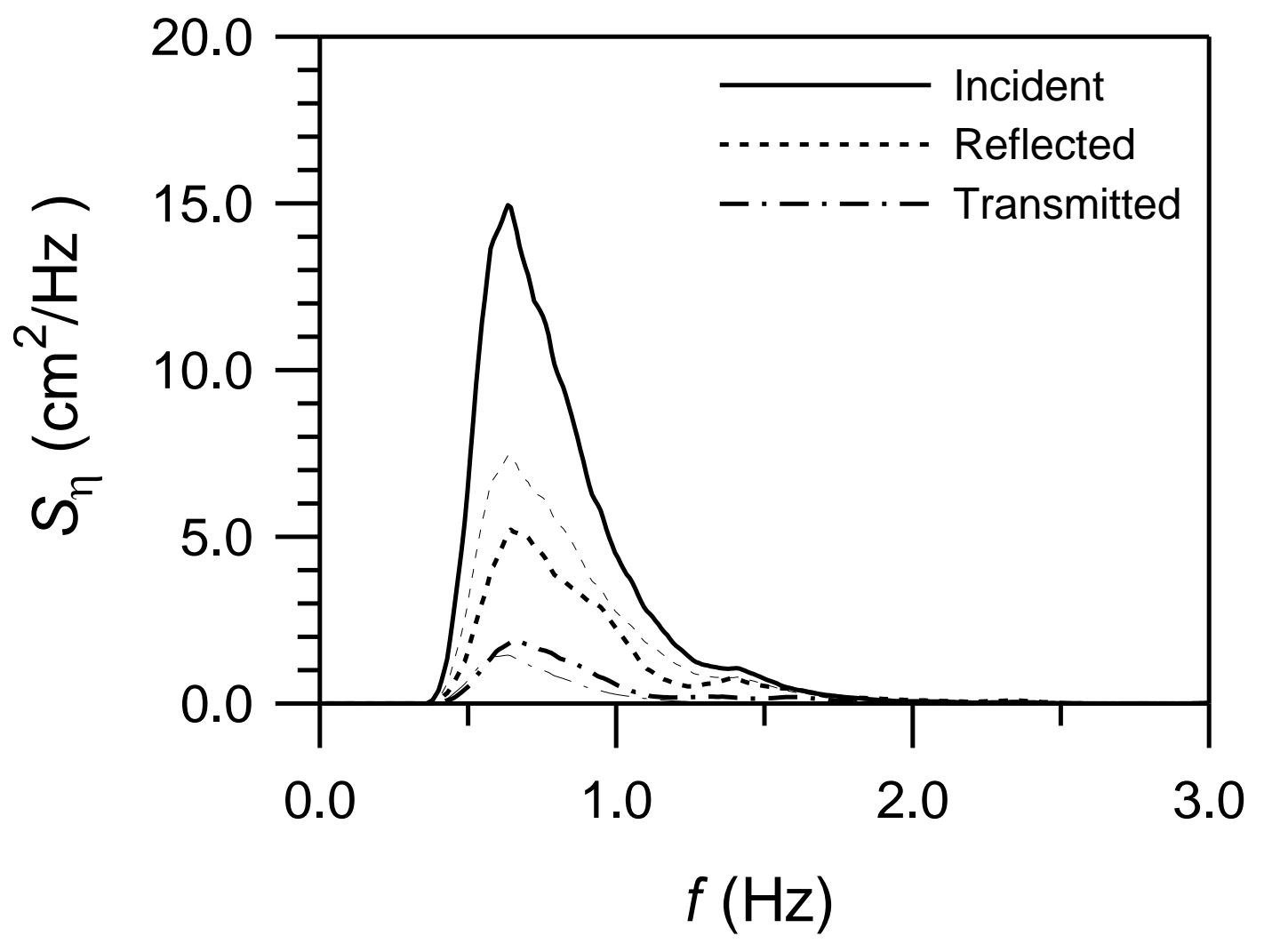

